\title{
Phase-field modelling of failure in hybrid laminates
}

\author{
R. Alessi ${ }^{a, *}$, F. Freddi ${ }^{b}$ \\ ${ }^{a}$ Department of Mathematics, Sapienza Università di Roma, Piazzale Aldo Moro 5, 00185 Roma, Italy \\ ${ }^{b}$ Department of Engineering and Architecture, University of Parma, Parco Area delle Scienze 181/A, I 43124 \\ Parma, Italy
}

\begin{abstract}
In this paper, the complex failure process of unidirectional hybrid laminates under uniaxial loading condition is reproduced and investigated by a one-dimensional phase-field model. The key ingredients of the approach, describing the mechanical response of a hybrid composite made of two different layers, are: (i) a phase-field method, based on a variational formulation of brittle fracture with regularised approximation of discontinuities for the two layers, (ii) cohesive law for the adhesive interface that connects the layers and (iii) robust and consolidated numerical strategy for the solution of the non-linear discretised problem. Explicit and well detailed simulations are shown for four peculiar failure mechanisms and the outcomes validated against experimental results available in literature. The model is able to discriminate among these different failure mechanisms according to the geometrical and mechanical properties of the hybrid composite. Both delamination of the adhesive interface is followed and crack patterns within the materials are fully determined. Finally, the proposed approach opens new perspective studies in higher dimension settings.
\end{abstract}

Keywords: Hybrid composites, Variational fracture, Cohesive Interface, Phase-field model

\section{Contents}

1 Introduction

2 The physical problem 5

3 The variational 1D model $\quad 6$

3.1 State variables, geometry and basic energetic quantities $\ldots \ldots \ldots \ldots$

3.2 Energetic formulation and evolution problem . . . . . . . . . . . . . 11

3.3 Evolution laws . . . . . . . . . . . . . . . . . . . . . . 12

4 Discrete problem and numerical implementation $\quad 12$

5 Numerical simulations and experimental validation $\quad 13$

6 Conclusion and perspectives $\quad 23$

Appendix A Construction of the evolution equations from the energy balance and the stability condition

\footnotetext{
${ }^{*}$ Corresponding Author

Email addresses: roberto.alessi@uniroma1.it (R. Alessi), francesco.freddi@unipr.it (F. Freddi)
} 


\section{Introduction}

Advanced lightweight applications such as aerostructures, spacecrafts, motorsports, high specification sports equipment and structural retrofitting are increasing the demand of high performance fibre reinforced composites, capable to guarantee high strength and stiffness together with low mass density. Unfortunately, these outstanding performances are matched with two main drawbacks: (i) high material and manufacturing costs and (ii) brittle failure without sufficient warning. In fact, unidirectional composite laminates are characterised by irreversible and brutal failure as the ultimate tensile stress is reached. In particular, unexpected failure and poor residual integrity prevents reliable and economical mass productions. The undesirable failure behaviour is usually overcome by conservative design limits, which inhibit designers from fully taking advantages of the superlative strength of composites. So, high performance composites that are characterised by a ductile or pseudo-ductile behaviour with predictable failsafe mechanisms similar to metals', where non-linear plastic strain hardening effects occur, are gaining popularity. In fact, research has focused attention to reach this peculiarity by the use of ductile [20] or tapered fibres [72], woven or braided fabrics [37, 40, 59] and more generically the use of hybrid materials $[1,60,26,28,70]$.

If properly designed, the failure process of a hybrid laminate involves a diffuse cracking (fragmentation) of the low-strain material, that is the material with the lowest ultimate strain, and possibly a diffuse delamination of the interface, Fig. 1a. Such failure process has the advantage to offer higher energy dissipation than in the case of sudden failure, presenting fibres and interface fractures dispersed in the entire composite which allow the composite to achieve a pseudo-ductile behaviour.

A similar fragmentation processes, due to non-local effects obtained by linking in parallel a stronger material with a weaker one, can be observed in many other different situations, as, for instance, in reinforced concrete elements Fig. 1b or fibre-reinforced materials Fig. 1c. Moreover, the mechanical behaviour is indirectly linked to other situations, as when the non-local effects due to material coupling are bypassed by an external prescribed strain, such as thermal shocks problems, Fig. 1d, or thin films failure, Fig. 1e. In addition, strong analogies can be also found in the mechanics of muscles, Fig. 1f.

A pseudo-ductile response has been obtained in thin-ply laminates by hybridised continuous fibres $[25,45,24]$. This behaviour has been achieved by properly combining two or more families of fibres such as carbon/glass fibres and by optimal designing the composite, specifically the thicknesses of each layer, with respect to the mechanical properties of the fibres.

The failure behaviour of the hybrid composite can be effectively characterised by the use of the so called Damage Mode Map (DMM), a 2D graph which defines four different failure regions as a function of the geometric layup properties, namely: (i) premature failure of the high-strain material, (ii) catastrophic delamination, (iii) multiple fractures of the low-strain material, (iv) fragmentation in the low-strain material followed by diffuse interface delamination. Experimental campaigns confirm the validity of such failure schematisation, [44, 45].

In the last years, numerical simulations were conducted in [44] to reproduce the behaviour of hybrid composite at the interface level. On the other side, an analytic model with a rigidperfectly plastic interface between the layers has been proposed in [45]. Ultimate tensile stress has been obtained from closed form solutions even if it underestimates the specific crack length and implies the use of stochastic tools to induce and predict the crack evolution in the low-strain layer [69].

A deep experimental campaign has been carried out in [43] where the interface behaviour is investigated. Starting from SEM images post-processed with digital image correlation, the authors observed the delamination process in fibre/epoxy composite materials. It has been evidenced that the energy is mainly dissipated through two mechanisms: creation of new fracture 


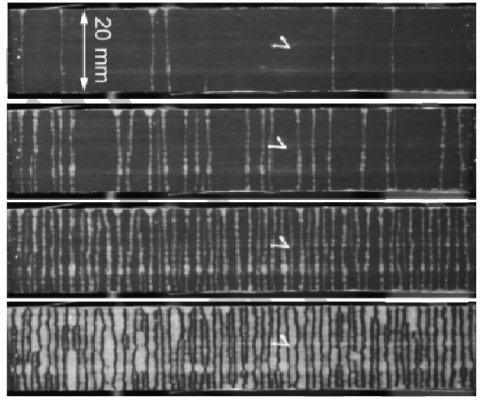

(a)

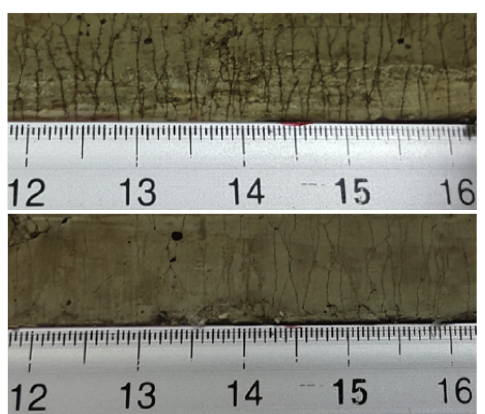

(c)

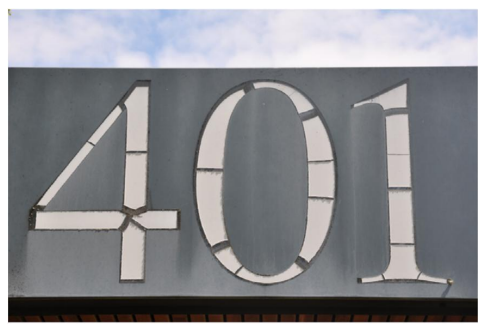

(e)
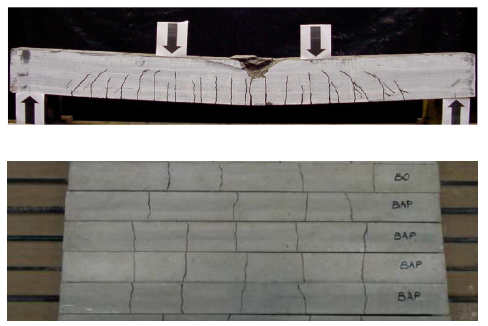

(b)

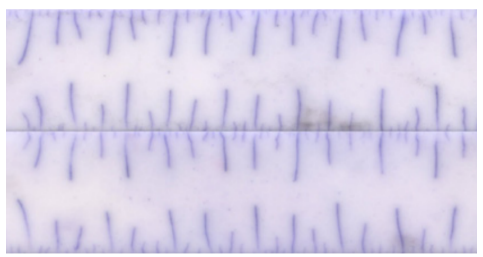

(d)

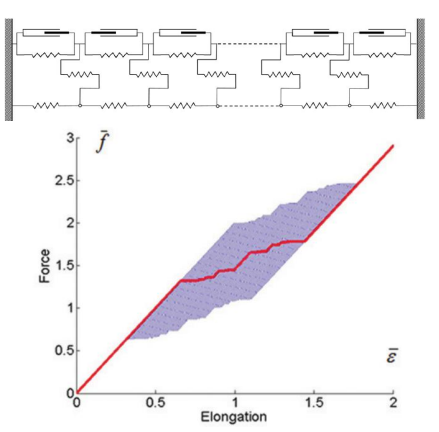

(f)

Figure 1: Examples of fragmentation or periodic fractures processes in different mechanical situations. (a) Progressive fragmentation and diffuse delamination in a hybrid composite laminate for a standard tensile test, [24]; (b) Crack patterns in a reinforced concrete beam under bending, [68], and in a reinforced concrete bar in traction, [22]; (c) Fragmentation in a ultra-high-ductile polyethylene-fibre-reinforced concrete after a standard tensile test [21]; (d) Upper and bottom side of a thin ceramic plate after a thermal shock, [46]; (e) Debonding of cracked lettering at École Polytechnique, Palaiseau, France, [48]; (f) Half-sarcomeres chain elastically linked to passive tissues, [61]. 
surfaces (delamination) and nonlinear shear deformations in the composite plies adjacent to the delaminating interface. Then, the nonlinear process zone is not limited to the interface between the layers, but also extends into the fibre/epoxy composite layers. Finally, an experimental trapezoidal traction-slip cohesive law at the interface is highlighted, inspiring and validating the interfacial law of the present work.

So far, to the authors knowledge, very few numerical tools are available for automatically capturing the failure processes of hybrid laminates, even in a simple 1D setup. An attempt is proposed in [53] where it has been demonstrated the ability of a non-local model to address localised deformation, strain softening and strength prediction of composite laminates. Such an instrument could be an efficient design tool which would permit, for instance, to determine the failure process, as the resulting fracture pattern and the dissipative capabilities of the hybrid composite, for a wide range of geometries, layups and loading conditions. With this longterm goal in mind, the authors propose in this work a novel variational phase-field model and a consistent numerical solution strategy, for 1D responses as a benchmark, setting the bases for future large scale computations involving more complex and generic cases. The fact that 1D models are fundamental and often sufficient for the understanding and the description of complex mechanical behaviours is widely accepted as testified by numerous works $[45,49,62,67]$ and many others.

In the last two decades, fracture problems have exploited advantages from the calculus of variations and $\Gamma$-convergence results $[10,29,27,16]$. The problem has been deeply studied, although still not exhaustively, and numerical effective tools have been setup taking advantage of a regularised version of the fracture problem.

The difficulties of a discrete crack approach in capturing crack nucleation, propagation, and bifurcation, and in general softening localisation phenomena, can be easily overcome by the so called phase-field model that have recently gained widespread popularity for simulating brittle crack problems in a smeared manner. The first original formulation [15] has been specified to prevent material overlapping and to reproduce particular failure modes due to specific material behaviour [11], [47], [32], [33] and [9] for a review. The debonding and fracture of a thin brittle film has been dealt with in [48, 73], Fig. 1e, while thermal shocks problems in [18], Fig. 1d. Moreover, dynamic fractures propagation has been investigated in [17, 52, 51, 14, 13]. More recently, extension to plastic material has been proposed in $[6,34,7,35,5,56]$ for the description of ductile and cohesive fractures, and first attempts to the study of cohesive material response can be found in [31, 23]. Finally, application to phase transformation localisations in shape memory alloys can be found in $[3,50,8]$.

On the other hand, for a thin adhesive layer connecting two solids, the kinematic of the interface is commonly defined by relative displacements (slips). Typically, the corresponding interface tangential stresses are linked to the slips by a non-linear relation (cohesive law), [36] for an exhaustive review.

The outline of the paper is the following. Firstly, the investigated problem is physically illustrated in Sec. 2. Subsequently, a variational formulation for the description of the mechanical response of a uni-directional (UD) hybrid laminate is proposed, Sec. 3. In Sec. 4, the discrete problem, based on an incremental minimisation scheme, is presented and the implementation strategy discussed. Then, Sec. 5 presents four numerical simulations, validated against experimental results, which are associated to the four typical failure modes. Finally, conclusions and perspectives are drawn in Sec. 6.

Concerning notation, unless otherwise specified, a prime $\square^{\prime}$ indicates either the derivative with respect to the spatial coordinate or the directional derivative of functionals; a superposed $\dot{\square}$ means right-derivative with respect to time. 


\section{The physical problem}

In this section, the investigated physical problem is presented. Specifically, the mechanical response, up to failure, of a hybrid UD composite laminate subjected to an in-plane monotonic imposed displacement along the fibre directions is studied. The hybrid laminate is obtained by combining three layers: the outer ones consisting of a high-strain elastic-brittle composite $(e$. $g$. glass fiber composite) whereas the middle one of a low-strain elastic-brittle composite $(e . g$. carbon fiber composite). The layers are connected through an adhesive interface that exhibits a nonlinear behaviour. Different interface laws among the layers can be considered. In general, these own an elastic stage and a dissipating phase, often softening, up to complete rupture corresponding to delamination. Configurations with more layers, alternating periodically the two materials, can be correlated to the response of the present setup, by assuming a homogeneous state along the thickness, [4, Fig. 1]. For symmetric reasons, which exclude bending effects, and since the length $L$ is assumed much longer than the thickness $h$, namely $h \ll L$, it is sufficient to study half the thickness of the laminate in a one-dimensional traction bar test setup, as represented in Fig. 2. Multidirectional laminates and general in-plane loadings, which obliged to consider at least two-dimensional geometries, will be investigated in a forthcoming work. Nevertheless, the present formulation can be easily extended to consider such general setting.

\section{physical problem}

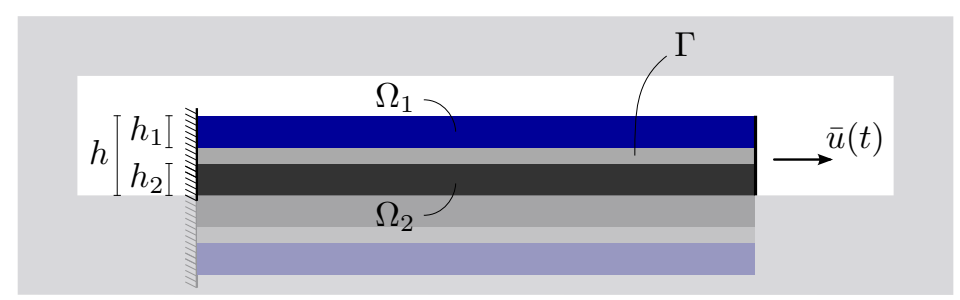

mathematical model

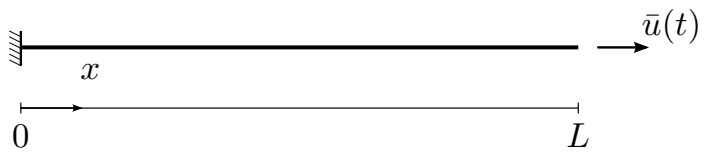

Figure 2: Physical problem and mathematical model, corresponding to a one-dimensional traction bar test setup. The interface $\Gamma$, represented with a finite thickness for sake of clarity, is actually of evanescent thickness.

After an initial elastic response, where the two layers experience the same homogeneous strain, a first fracture in the low-strain layer always occur. Then, the mechanical response of the fractured laminate changes abruptly and by further increasing the bar end displacement, four different failure mechanisms are possible, namely

- Premature failure of the high-strain layer by a fracture spanning the entire laminate thickness, extended instantaneously from the first low-strain layer crack $(\mathcal{P})$;

- Catastrophic delamination covering almost all the laminate and subsequent failure of the high-strain layer $(\mathcal{C})$;

- Progressive multi-cracking (fragmentation) of the low-strain layer without interface delamination up to the failure of the high-strain layer $(\mathcal{F})$;

- Progressive fragmentation of the low-strain layer and diffuse delamination of the interface up to the failure of the high-strain layer leading to the pseudo-ductile response $(\mathcal{D})$. 
These different failure modes are visualised in Fig. 3 with the corresponding qualitative forcedisplacement responses.

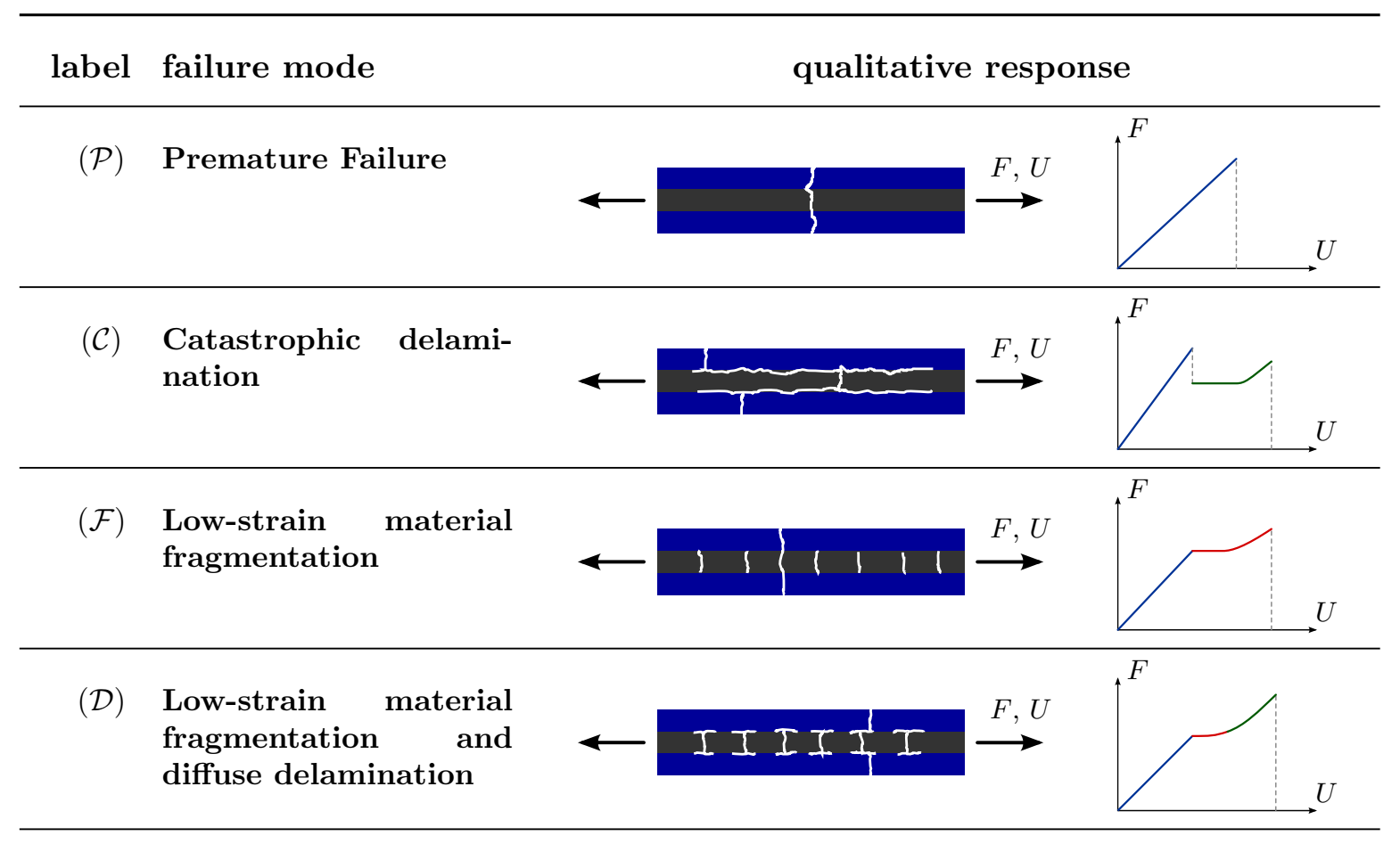

Figure 3: Different failure modes of the UD hybrid laminate. The blue, green and red curves in the qualitative global responses refer, respectively, to the elastic, fragmentation and diffuse delamination stages.

The occurrence of these failure mechanisms is strongly related to the mechanical properties of the materials and to the geometric parameters of the composite laminate. For given materials, the variability of the failure mechanisms with respect to the layer thicknesses can be effectively represented in the, so called, damage mode map (DMM) as in [44, 4].

\section{The variational $1 \mathrm{D}$ model}

The one-dimensional continuum model, illustrated in the previous section, Fig. 2, consists of two layers with thickness $h_{1}$ and $h_{2}$, respectively, bonded together by a cohesive interface $\Gamma$, whose thickness is assumed very thin compared to $h_{1}$ and $h_{2}$. The first layer $\Omega_{1}$, denominated high-strain layer, has a higher failure strain compared to the second layer $\Omega_{2}$, therefore called low-strain layer. The hybrid bar, of length $L$, is subjected to a prescribed time-dependent displacement $\bar{u}(t)$ applied to the right-end side, whereas the left-end side is fixed such to fulfil the standard traction bar test setup. The applied displacement is assumed to be monotonically increasing at a sufficiently low rate; as such, rate-dependent and inertial effects can be neglected from the analysis.

The high- and low-strain layers are assumed to have a linear elastic-brittle response with tensile moduli $\overline{\mathrm{E}}_{1}, \overline{\mathrm{E}}_{2}$ and ultimate strains $\bar{\varepsilon}_{1}, \bar{\varepsilon}_{2}$, respectively, Fig. 4. Correspondingly, the ultimate stresses are $\bar{\sigma}_{1}:=\overline{\mathrm{E}}_{1} \bar{\varepsilon}_{1}$ and $\bar{\sigma}_{2}:=\overline{\mathrm{E}}_{2} \bar{\varepsilon}_{2}$.

In the present approach, the brittle-fracture behaviour of the two layers is modelled by means of a regularised variational model (often called phase-field approach). The capability and effectiveness of this strategy to reproduce brittle-fracture phenomena is nowadays quite consolidated, [55]. The variational model of quasi-static crack evolution is based on the minimisation of an energy functional composed of a bulk term, associated to the elastic energy of the 


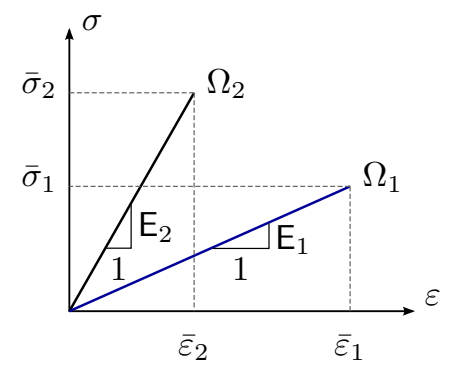

Figure 4: Mechanical response of the two single layers.

material, and a surface term, associated to the Griffith's fracture dissipated energy. Therefore, the unknowns of the problem are the macroscopic displacement field and the fracture path, this last leading to a hard solvable free-discontinuity problem, [19].

The key idea behind the regularised version of this fracture problem, which is much more feasible to be solved, is the replacement of the unknown fracture path with an additional field, often named phase field, representing the damage state of the material. In the present context, the phase field varies between 0 and 1 , specifically unity value in cracked zones and zero in a sound one.

Furthermore, the spatial gradient of the phase field directly affects the energy value of the functional. Therefore, the regularised functional is also characterised by an internal auxiliary parameter having the dimension of a length that defines the size of the transition zone between sound and broken portions of the material.

Concerning the interface, a suitable cohesive law, that relates shear stress and displacement slip between the layers, is derived from a potential, in accordance with experimental evidences [43].

\subsection{State variables, geometry and basic energetic quantities}

In the present one-dimensional setting, we assume that each point $x \in[0, L]$ of the bar is characterised by the state variables described in Tab. 1. Such variables are therefore assumed to be constant along the thickness of each layer. Moreover, according to experimental evidences, small-strain regime is assumed. The bar is considered fixed at the left extremity and an external

\begin{tabular}{cll}
\hline domain & state variable \\
\hline \multirow{3}{*}{$\Omega_{i}$} & $u_{i}$ & displacement \\
$(i=1,2)$ & $\varepsilon_{i}$ & infinitesimal total strain \\
& $\alpha_{i}$ & damage (irreversible) \\
& $\gamma_{i}$ & gradient damage \\
\hline \multirow{2}{*}{$\Gamma$} & $\delta$ & slip \\
& $\delta_{\mathrm{c}}$ & maximum slip (history variable) \\
\hline
\end{tabular}

Table 1: State variables.

displacement is prescribed at the right-end

$$
u_{1}(0, t)=u_{2}(0, t)=0 \quad \text { and } \quad u_{1}(L, t)=u_{2}(L, t)=\bar{u}(t)
$$

with $\bar{u}(0)=0$ and $t$ being a time-like parameter, in accordance with the setup depicted in Fig. 2. Without loss of generality, the applied displacement is assumed to be smooth (at least 
continuous) such to comply with a quasi-static setting.

The corresponding time-dependent state variable fields are labelled with the same letters and only the displacement field $u(x, t)$ and the damage field $\alpha(x, t)$ of the two layers are truly independent. Indeed, the infinitesimal strain, gradient of damage and the slip are given by

$$
\varepsilon_{i}(x, t)=u_{i}^{\prime}(x, t), \quad \gamma_{i}(x, t)=\alpha_{i}^{\prime}(x, t), \quad \delta(x, t)=u_{1}(x, t)-u_{2}(x, t)
$$

with $i=1,2$. The maximum slip is a history variable, defined as

$$
\delta_{\mathrm{c}}(x, t):=\max _{\tau \in[0, t]}|\delta(x, \tau)|
$$

The scalar damage fields are bounded, since we assume

$$
\alpha_{i} \in[0,1] \quad \text { with } \quad \begin{cases}\alpha_{i}=0 & \text { sound material } \\ \alpha_{i}=1 & \text { fully damaged material }\end{cases}
$$

and must satisfy the following irreversibility conditions

$$
\dot{\alpha}_{i} \geq 0, \quad \text { on }[0, L] \text { with } i=1,2 \text { and } \forall t
$$

since no healing effects are taken into account within the material.

The composite bar is initially $(t=0)$ undeformed, undamaged and the interface is fully sound.

Once the internal variables have been set and according to the energetic formulation [58], it is possible to introduce the basic energetic quantities on which the entire evolution of the system relies on. This task is effectively accomplished in the framework of Generalized Standard Materials, $[42,57,2]$. In such a setting, we assume the internal potential energy density as the sum of three contributions

$$
\psi\left(\varepsilon_{1}, \alpha_{1}, \gamma_{1}, \varepsilon_{2}, \alpha_{2}, \gamma_{2}, \delta, \delta_{\mathrm{c}}\right):=h_{1} \psi_{1}\left(\varepsilon_{1}, \alpha_{1}, \gamma_{1}\right)+h_{2} \psi_{2}\left(\varepsilon_{2}, \alpha_{2}, \gamma_{2}\right)+\psi_{\Gamma}\left(\delta, \delta_{\mathrm{c}}\right)
$$

with $\psi_{1}$ and $\psi_{2}$ being the internal elastic potential energy density of, respectively, layer 1 and layer 2 , whereas $\psi_{\Gamma}$ being the internal energy density of the interface. Specifically, in the present infinitesimal strain setting, we have,

$$
\psi_{i}\left(\varepsilon_{i}, \alpha_{i}, \gamma_{i}\right):=\frac{1}{2} \mathrm{E}_{i}\left(\alpha_{i}\right) \varepsilon_{i}^{2}+\frac{1}{2} \overline{\mathrm{w}}_{i} \eta_{i}^{2} \gamma_{i}^{2}, \quad \psi_{\Gamma}\left(\delta, \delta_{\mathrm{c}}\right):= \begin{cases}\psi_{\Gamma}(\delta), & \text { if } \delta_{\mathrm{c}} \leq \bar{\delta} \\ 0, & \text { if } \delta_{\mathrm{c}}>\bar{\delta}\end{cases}
$$

where $\mathrm{E}_{i}\left(\alpha_{i}\right)$ represent the Young elastic moduli and are supposed to decrease as damage evolves, [55]. Specifically, the functions $\alpha_{i} \mapsto \mathrm{E}_{i}\left(\alpha_{i}\right)$ are continuously differentiable on $[0,1)$ and fulfil the following conditions

$$
\mathrm{E}_{i}(0)=\overline{\mathrm{E}}_{i} \quad \mathrm{E}_{i}^{\prime}(0)<0, \quad \mathrm{E}_{i}^{\prime}\left(\alpha_{i}\right) \leq 0, \quad \mathrm{E}_{i}(1)=0
$$

with $\overline{\mathrm{E}}_{i}$ being the elastic Young moduli of the sound materials. The small quantities $\eta_{i}$ are internal material lengths which determine the width of the damage transition between damaged and undamaged state in each layer. As $\eta_{i} \rightarrow 0$, the damage profile tends to represent a sharp fracture. The constants $\overline{\mathrm{w}}_{i}$ are related to the damage dissipation, which is later defined in (3.12). 


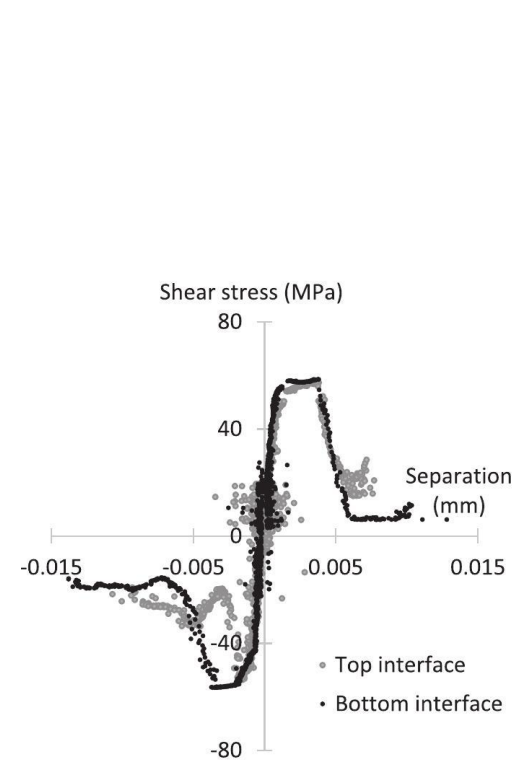

(a)

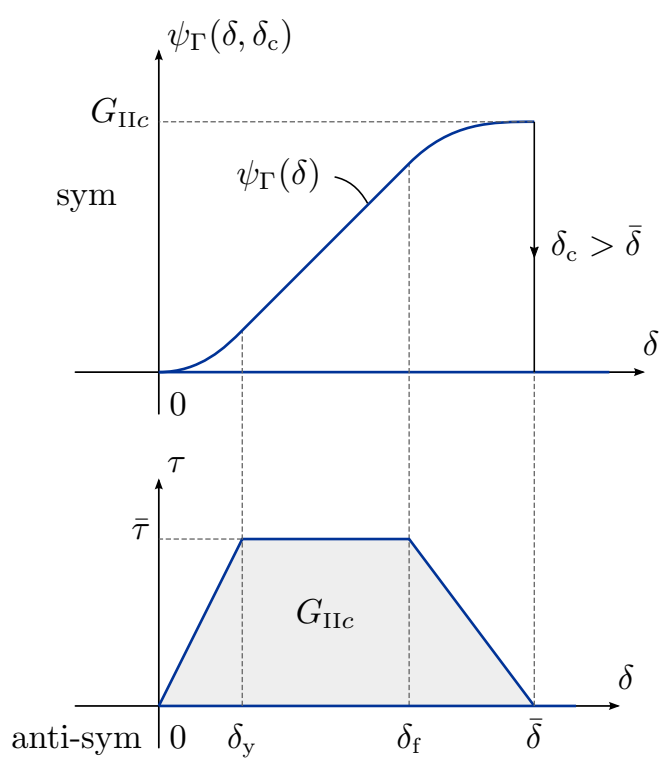

(b)

Figure 5: (a) Experimental results concerning the shear stress-slip relation of an interface in a hybrid glass/carbon UD laminate. Picture and results are taken from [43]. (b) Interface potential and respective tri-linear $\tau-\delta$ diagram used in the numeric simulations in Sec. 5 .

From the internal potential energy density we define the stresses, as

$$
\sigma_{i}:=\partial_{\varepsilon_{i}} \psi_{i}=\mathrm{E}_{i}\left(\alpha_{i}\right) \varepsilon_{i}, \quad \tau:=\partial_{\delta} \psi_{\Gamma}\left(\delta, \delta_{\mathrm{c}}\right)= \begin{cases}\psi_{\Gamma}^{\prime}(\delta), & \text { if } \delta_{\mathrm{c}} \leq \bar{\delta} \\ 0, & \text { if } \delta_{\mathrm{c}}>\bar{\delta}\end{cases}
$$

being $\bar{\delta}$ the limit slip which triggers delamination.

As an example, we represent in Fig. $5 \mathrm{~b}$ the interface internal potential $\psi_{\Gamma}\left(\delta, \delta_{\mathrm{c}}\right)$ and the respective tri-linear stress law used, later on, in the numeric simulations. Physical justification of this assumption is give in Section 5 .

Accordingly, the total internal potential energy reads

$$
\begin{aligned}
\mathcal{E}\left(u_{1}, \alpha_{1}, u_{2}, \alpha_{2}, \delta_{\mathrm{c}}\right)= & \int_{0}^{L} \psi\left(\varepsilon_{1}, \alpha_{1}, \gamma_{1}, \varepsilon_{2}, \alpha_{2}, \gamma_{2}, \delta, \delta_{\mathrm{c}}\right) \mathrm{d} x= \\
& =\int_{0}^{L}\left(h_{1} \psi_{1}\left(u_{1}^{\prime}, \alpha_{1}, \alpha_{1}^{\prime}\right)+h_{2} \psi_{2}\left(u_{2}^{\prime}, \alpha_{2}, \alpha_{2}^{\prime}\right)+\psi_{\Gamma}\left(u_{1}-u_{2}, \delta_{\mathrm{c}}\right)\right) \mathrm{d} x
\end{aligned}
$$

The total dissipated work up to an instant $t$ is, in general, a process dependent function. Nevertheless, due to the underlying gradient damage model for which the dissipated work is a state function and due to the simplified modeling of the interface law, the dissipated work density becomes a state function, sum of three contributions

$$
\varphi\left(\alpha_{1}, \alpha_{2}, \delta_{\mathrm{c}}\right):=h_{1} \varphi_{1}\left(\alpha_{1}\right)+h_{2} \varphi_{2}\left(\alpha_{2}\right)+\varphi_{\Gamma}\left(\delta_{\mathrm{c}}\right)
$$

with $\varphi_{1}$ and $\varphi_{2}$ being the dissipated work density of, respectively, layer 1 and layer 2 while $\varphi_{\Gamma}$ 
being the dissipated work of the interface. Specifically, we have

$$
\varphi_{i}\left(\alpha_{i}\right):=\mathrm{w}_{i}\left(\alpha_{i}\right), \quad \varphi_{\Gamma}\left(\delta_{\mathrm{c}}\right):= \begin{cases}0, & \text { if } \delta_{\mathrm{c}} \leq \bar{\delta} \\ G_{\mathrm{II} c}, & \text { if } \delta_{\mathrm{c}}>\bar{\delta}\end{cases}
$$

this last chosen in order to be equal to the area underneath the shear stress-slip curve (Fig. 5b), namely

$$
G_{\mathrm{II} c}:=\int_{0}^{\bar{\delta}} \partial_{\delta} \psi_{\Gamma}\left(\delta, \delta_{\mathrm{c}}\right) \mathrm{d} \delta=\varphi_{\Gamma}(\bar{\delta})
$$

The quantity $G_{\text {IIc }}$ represents the mode-II fracture toughness of the interface. According to standard gradient damage models, [65], the damage functions $w_{i}(\alpha)$, representing the energy dissipated in the damage process in each layer, satisfy the following conditions

$$
\mathrm{w}_{i}(0)=0, \quad \mathrm{w}_{i}^{\prime}(\alpha) \geq 0, \quad \mathrm{w}_{i}^{\prime}(1)=\overline{\mathrm{w}}_{i}
$$

The risk for shear strain localisations at the interface level is precluded by the presence of two different layers continuously connected in parallel. In fact, the high-strain layer, which remains sound up to the complete failure, induces a non-local effect on the interface that interacts with the other layer. Such non-local effect is very similar to the one described in [61,62], concerning a half sarcomeres chain surrounded by a connecting tissue and modelled by two non-linear parallel elastic chains connected with cross linear elastic springs.

The corresponding total dissipated work then reads

$$
\mathcal{D}\left(\alpha_{1}, \alpha_{2}, \delta_{\mathrm{c}}\right):=\int_{0}^{L} \varphi\left(\alpha_{1}, \alpha_{2}, \delta_{\mathrm{c}}\right) \mathrm{d} x=\int_{0}^{L}\left(h_{1} \varphi_{1}\left(\alpha_{1}\right)+h_{2} \varphi_{2}\left(\alpha_{2}\right)+\varphi_{\Gamma}\left(\delta_{\mathrm{c}}\right)\right) \mathrm{d} x
$$

It is worth to remark again that for the present phase-field model, the resulting dissipated work is a state-dependent function and not, as usual, a path-dependent quantity.

The interaction between the system and the surrounding environment is characterised by the external power, here only due to prescribed displacements. In particular, since the displacement of one bar end is always kept fixed for both layers and since only the displacement of the other bar end is prescribed, (3.1), the total external power reads

$$
\mathcal{L}(t)=\int_{0}^{t}\left(f_{1}(t)+f_{2}(t)\right) \dot{\bar{u}}(t) \mathrm{d} \tau
$$

with $f_{1}(t)$ and $f_{2}(t)$ being, respectively, the unknown reaction forces of the two layers.

Remark 3.1 (Specific model). As phase-field model for the description of the fracture process for both layers, we chose Example 1 in [55], which owns an explicit elastic stage. For such a model

$$
\mathrm{E}(\alpha)=\overline{\mathrm{E}}(1-\alpha)^{2} \quad \text { and } \quad \mathrm{w}(\alpha)=\overline{\mathrm{w}} \alpha
$$

The ultimate stress, the amplitude of the localisation and the fracture toughness are given by

$$
\bar{\sigma}=\sqrt{\overline{\mathrm{E}} \overline{\mathrm{w}}}, \quad 2 D=2 \sqrt{2} \eta, \quad G_{c}=\frac{8}{3 \sqrt{2}} \overline{\mathrm{w}} \eta
$$

as a result of the damage localization analysis and its link with Griffith's brittle fracture model, [55, Sec. 3.3.2].

Concerning the $\tau-\delta$ interface law, recent studies ([43] and Fig. 5a) suggest to use, in the 
present context of hybrid laminates, a tri-linear law, namely

$$
\psi_{\Gamma}^{\prime}(\delta)= \begin{cases}K \delta, & \text { if }|\delta| \leq \delta_{\mathrm{y}} \\ \bar{\tau}, & \text { if } \delta_{\mathrm{y}}<|\delta| \leq \delta_{\mathrm{f}} \\ \operatorname{sign}(\delta)\left(\frac{|\delta|-\bar{\delta}}{\delta_{\mathrm{f}}-\bar{\delta}}\right) \bar{\tau}, & \text { if } \delta_{\mathrm{f}}<|\delta|(\leq \bar{\delta})\end{cases}
$$

with $\bar{\tau}:=K \delta_{\mathrm{y}}$ and $K$ being the interface elastic stiffness. Such a law is represented in Fig. $5 b$ with its related potential.

\subsection{Energetic formulation and evolution problem}

According to the energetic formulation, the evolution of a rate-independent system is governed by three energetic principles: an energy balance, a dissipation inequality and a stability criterion, $[57,58]$. Moreover, some explicit irreversibility conditions may by prescribed, as in the present context for the damage field, (ir). While energy balance and the dissipation inequality are nothing but particular statements of the first and second law of thermodynamics, they are, in general, not sufficient to characterise the solution process and further restrictions, given by the stability criterion, are necessary, [2].

For the purposes of the present work and exploiting the regularity of the energetic functionals, it is sufficient to consider the first-order energy balance condition, derived from the global condition by expanding in time the energetic terms, and the first-order (local directional) stability condition. The reader can refer to $[65,67,3,2,8]$ for further details as, for instance, higher order differential energy balance and stability conditions ${ }^{1}$.

In the present context, a process $\left(u_{1}, \alpha_{1}, u_{2}, \alpha_{2}, \delta_{\mathrm{c}}\right)_{t}$ satisfies the first-order energy balance in a time interval $[0, T]$ if

$$
\frac{\mathrm{d}}{\mathrm{d} t}\left(\mathcal{E}\left(u_{1}, \alpha_{1}, u_{2}, \alpha_{2}, \delta_{\mathrm{c}}\right)+\mathcal{D}\left(\alpha_{1}, \alpha_{2}, \delta_{\mathrm{c}}\right)-\mathcal{L}(t)\right)=0, \quad \forall t \in[0, T]
$$

A process $\left(u_{1}, \alpha_{1}, u_{2}, \alpha_{2}, \delta_{\mathrm{c}}\right)_{t}$ is said to satisfy the dissipation inequality in a time interval $[0, T]$ if the dissipated power at any point is non-negative, namely

$$
\frac{\mathrm{d}}{\mathrm{d} t} \varphi\left(\alpha_{1}, \alpha_{2}, \delta_{\mathrm{c}}\right) \geq 0, \quad \forall t \in[0, T]
$$

which clearly implies $\frac{\mathrm{d}}{\mathrm{d} t} \mathcal{D}\left(\alpha_{1}, \alpha_{2}, \delta_{\mathrm{c}}\right) \geq 0$. In the present context, (di) is automatically satisfied thanks to the irreversibility conditions (ir) and constitutive assumptions (3.12).

A process $\left(u_{1}, \alpha_{1}, u_{2}, \alpha_{2}, \delta_{\mathrm{c}}\right)_{t}$ satisfies the first-order stability condition in a time interval $[0, T]$ if for any admissible test direction $\left(\widetilde{u}_{1}, \widetilde{\alpha}_{1}, \widetilde{u}_{2}, \widetilde{\alpha}_{2}\right)$ one has

$$
\mathcal{E}^{\prime}\left(u_{1}, \alpha_{1}, u_{2}, \alpha_{2}, \delta_{\mathrm{c}}\right)\left(\widetilde{u}_{1}, \widetilde{\alpha}_{1}, \widetilde{u}_{2}, \widetilde{\alpha}_{2}, 0\right)+\mathcal{D}^{\prime}\left(\alpha_{1}, \alpha_{2}, \delta_{\mathrm{c}}\right)\left(\widetilde{\alpha}_{1}, \widetilde{\alpha}_{2}, 0\right) \geq 0, \quad \forall t \in[0, T]
$$

with $\mathcal{E}^{\prime}$ and $\mathcal{D}^{\prime}$ being the standard Gâteaux derivatives of the potential energy and dissipated work, respectively.

\footnotetext{
${ }^{1}$ The first-order stability condition is necessary but, in general, not sufficient in order to satisfy the full local directional stability condition at each time instant of the evolution, [3, 2].
} 


\subsection{Evolution laws}

The equations that govern the problems are listed in Tab. 2, consisting of two coupled equilibrium equations and two uncoupled sets of Karush-Kunt-Tucker (KKT) conditions driving the evolution of the phase fields, all of them completed with proper boundary conditions. It is worth to remark that these evolution laws have been variationally (and automatically) deduced from the energetic formulation (energy balance + stability) and not a priori prescribed.

layer equilibrium conditions damage conditions

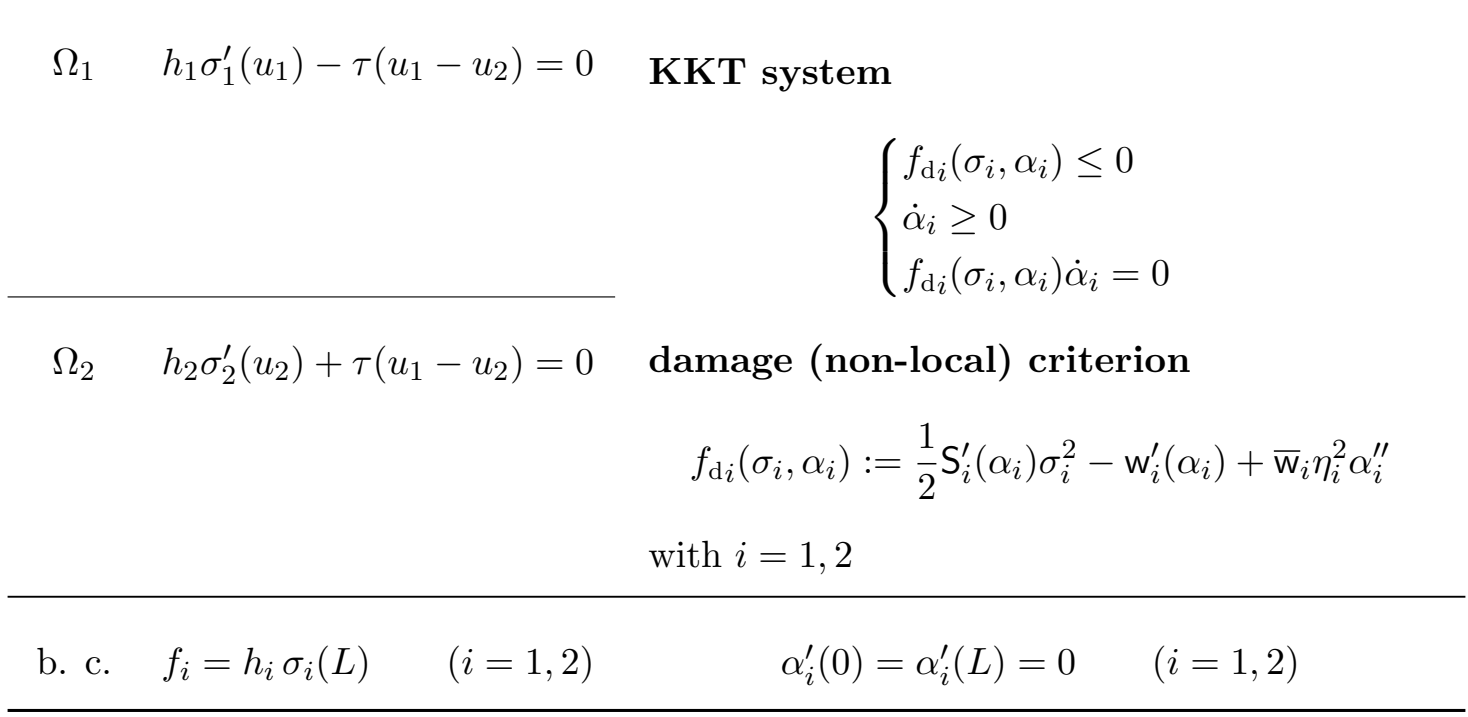

Table 2: Summary of the governing equations deduced from the first-order energy balance (eb) and the first-order stability condition (st). In $f_{\mathrm{d}}, \mathrm{S}(\alpha):=\mathrm{E}^{-1}(\alpha)$ represents the elastic compliance of each layer.

\section{Discrete problem and numerical implementation}

Energy balance and stability turn into an incremental energy minimisation problem, [64]. For the present model, since the dissipated work is a state function, apart for the history variable $\delta_{\mathrm{c}}$, a total internal energy $\mathcal{W}$ (or total internal strain work) can be introduced, [66], namely

$$
\mathcal{W}\left(u_{1}, \alpha_{1}, u_{2}, \alpha_{2}, \delta_{\mathrm{c}}\right):=\mathcal{E}\left(u_{1}, \alpha_{1}, u_{2}, \alpha_{2}, \delta_{\mathrm{c}}\right)+\mathcal{D}\left(\alpha_{1}, \alpha_{2}, \delta_{\mathrm{c}}\right)
$$

Since no external loadings are considered, but only applied displacements, the (discrete) incremental minimisation problem then simply becomes

$$
\begin{aligned}
\left(u_{1}, \alpha_{1}, u_{2}, \alpha_{2}\right)_{t_{i}}=\underset{u_{1}, \alpha_{1}, u_{2}, \alpha_{2}}{\arg \min } \mathcal{W}\left(u_{1}, \alpha_{1}, u_{2}, \alpha_{2}, \delta_{\mathrm{c}}\right) \\
\text { constrained to }\left\{\begin{array}{l}
u_{1}(0)=u_{2}(0)=0 \\
u_{1}(L)=u_{2}(L)=t_{i} \\
\left(\alpha_{1}\right)_{t_{i-1}} \leq \alpha_{1} \leq 1 \\
\left(\alpha_{2}\right)_{t_{i-1}} \leq \alpha_{2} \leq 1 \\
\delta_{\mathrm{c}}=\max \left(\left(\delta_{\mathrm{c}}\right)_{t_{i-1}},\left|u_{1}-u_{2}\right|\right)
\end{array}\right.
\end{aligned}
$$

The problem is numerically solved using an incremental procedure similar to that proposed in [15] where at each time step $i$ the numerical scheme is based upon an alternate minimisation 
algorithm which, in short, consists in solving a series of minimisation sub-problems on $u_{1,2}$ at fixed $\alpha_{1,2}$ and vice versa on $\alpha_{1,2}$ at fixed $u_{1,2}$, up to convergence. In particular, a Newton-like algorithm has been developed to detect the equilibrium for $\left(u_{1}, u_{2}\right)_{i}$ at each time step whereas the minimisation on $\alpha_{i}$ is obtained as the solution of two uncoupled quadratic constrained optimisation problems. The algorithm exploits the convexity with respect to each variable of the total energy (4.1). The code has been written using the FEniCS library [54] for finite elements and PETSc [12] for linear algebra operations, including bound-constrained solvers. Moreover, in the numerical simulations, the domain are discretised with uniformly distributed straight 1D elements with linear shape functions.

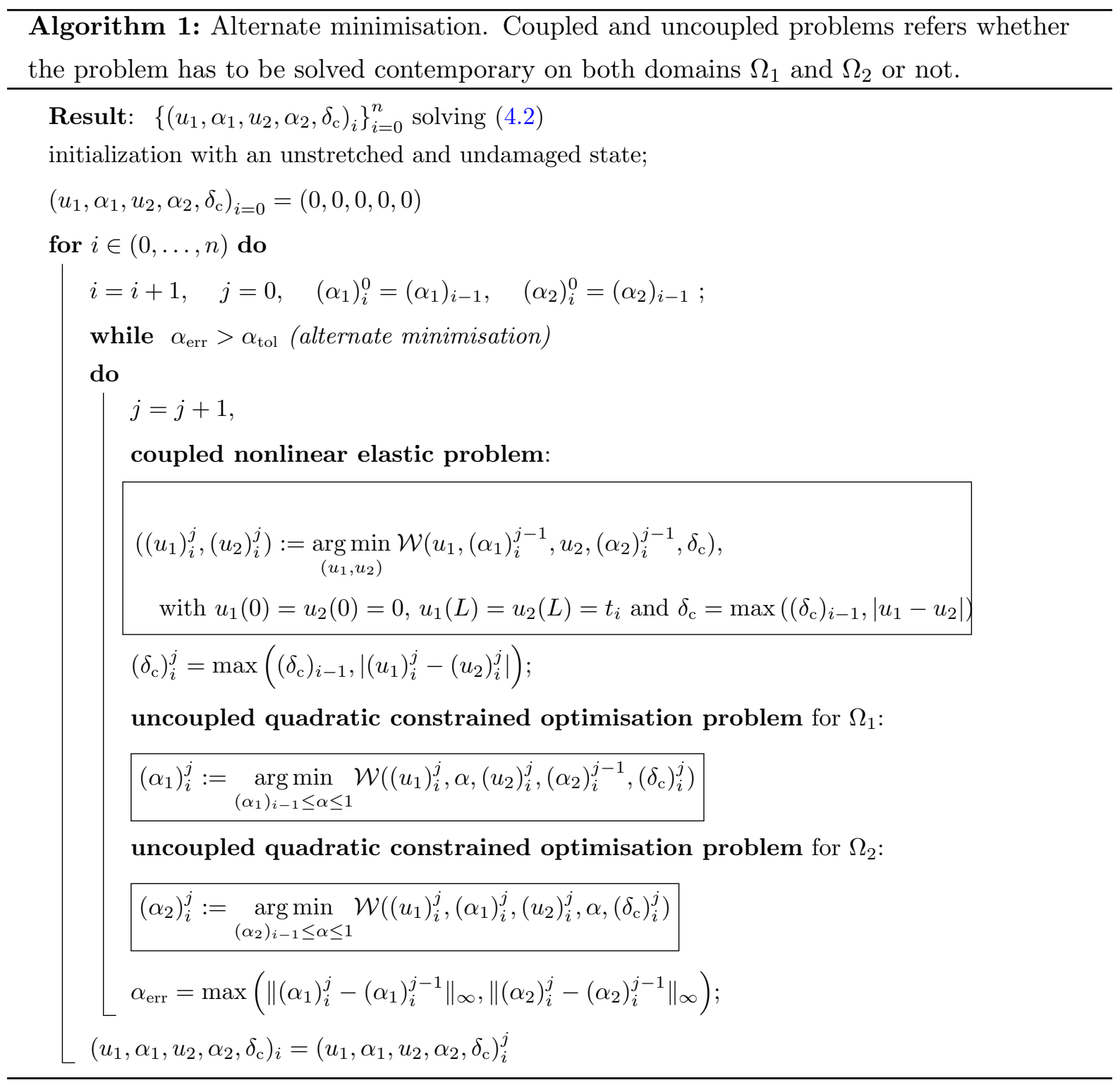

\section{Numerical simulations and experimental validation}

In this section, four numerical examples are presented, each of them referring to a different peculiar failure mode in Tab. 3, and the corresponding results validated against the experiments 
performed in $[25,45]$. These tests refer to four hybrid configurations, made of two different glass-epoxy/carbon-epoxy hybrid combinations.

Specifically, three basic layers are considered, two glass epoxy pre-impregnated composites (Hexcel E-glass/913 epoxy resin (EG) and Hexcel S-glass/913 epoxy resin (SG)) and a thin carbon epoxy composite (SkyFlex TR30). Their mechanical properties are summarised in Tab. 3.

\begin{tabular}{|c|c|c|c|c|c|c|}
\hline pre-impregnated composites & label & $\begin{array}{c}\mathrm{E} \\
(\mathrm{GPa})\end{array}$ & $\begin{array}{c}\bar{\sigma} \\
(\mathrm{MPa})\end{array}$ & $\begin{array}{c}\bar{\varepsilon} \\
(\%)\end{array}$ & $\begin{array}{c}\overline{\mathrm{w}}^{2} \\
(\mathrm{MPa})\end{array}$ & $\begin{array}{c}h \\
(\mathrm{~mm})\end{array}$ \\
\hline Hexcel E-Glass/913 epoxy resin, $\left(\Omega_{1}\right)$ & EG & 38.7 & 1548 & 4.00 & 61.9 & 0.144 \\
\hline Hexcel S-Glass/913 epoxy resin, $\left(\Omega_{1}\right)$ & $\mathrm{SG}$ & 45.7 & 2138 & 4.68 & 100.0 & 0.155 \\
\hline SkyFlex TR30 carbon epoxy, $\left(\Omega_{2}\right)$ & $\mathrm{C}$ & 101.7 & 2339 & 2.29 & 53.8 & 0.030 \\
\hline
\end{tabular}

Table 3: Material properties of E-glass ([41]), S-glass and TR30 carbon ([39]) pre-impregnated composites taken from [45].

According to [63], a random strength distribution based on Weibull's statistics is introduced for the low-strain layer. The actual strength of $\Omega_{2}$ is therefore assumed as

$$
\bar{\sigma}_{2}^{*}=\bar{\sigma}_{2}\left(\ln \left(\frac{1}{1-\lambda}\right)\right)^{1 / m}
$$

with $\bar{\sigma}_{2}$ and $m=29.3$ being, respectively, the Weibull shape and modulus parameters, and $\lambda$ a random variable ranging from 0 to 1 . In the present work, $\bar{\sigma}_{2}$ is the minimal expected strength for each layup, directly extrapolated from the experiments. Due to the lack of information and for the aims of the present work, a more complete and precise expression, taking into account size effects, is not provided. In addition, a lower bound for the variability of $\lambda$ is prescribed, precisely $\lambda_{\min } \simeq 0.00074: \ln \left(1 /\left(1-\lambda_{\min }\right)\right)=1$, which ensures that $\bar{\sigma}_{2}^{*} \geq \bar{\sigma}_{2}$.

Instead, a size-effect for the strength of the high-strain layer is taken into account in addition to Weibull's statistics, by adopting the following expression for the limit stress value

$$
\bar{\sigma}_{1}^{*}=\frac{\bar{\sigma}_{1}}{(1+\beta) V^{1 / m}}\left(\ln \left(\frac{1}{1-\lambda}\right)\right)^{1 / m}
$$

with $\beta=h_{2} / h_{1}, V$ being the whole volume of the low-strain material and $m=41$.

Numerically, the strength variability of both the low-strain and high-strain layers is obtained by changing accordingly the damage dissipation constant $\overline{\mathrm{w}}$, noting that

$$
\overline{\mathrm{w}}_{i}^{*}=\left(\bar{\sigma}_{i}^{*}\right)^{2} / \overline{\mathrm{E}}_{i}
$$

Deeper and more rigorous statistical analyses, affecting also other constitutive variables, are out of scope in the present work where, instead, the attention is more focused on the variational modeling and numerical implementation of the problem. For the same reasons, stress intensification effects around the interlaminar crack tip are not explicitly considered.

Eqs. (5.1) and (5.2) induce a strength perturbation on the overall composite and break the symmetry of the problem along the bar length. Nevertheless, it is worth pointing out that

\footnotetext{
${ }^{2}$ deduced from $(3.16)$
} 
the ultimate crack distances, in case of fragmentation of the low-strain layer, are only weakly affected by such perturbations, [45, 4].

The mechanical properties of the interface, not affected by statistical effects, have been deduced from the experimental results done in [43] and are summarised in Tab. 4.

The shear strength $\bar{\tau}$ has been obtained from $\pm 45^{\circ}$ shear tests carried out by [39] whereas the interface law and the limit slips are deduced from the recent experiments in [43, Fig. 11, reported also in the present work in Fig. 5a]. The interlaminar fracture toughness $G_{\text {IIc }}$ of the glass/carbon interface has been determined through a different experimental setup ensuring a stable crack propagation, $[71,25]$. Such tests unveil that the interlaminar fracture occurs in mode II, since the normal stress among the layers across the interface around the crack tip is compressive when the specimen is in tension. As pointed out in [43], the considered fracture toughness is three times greater, or even more, than the area underneath the true tractionseparation curve, since it also accounts for inelastic dissipative phenomena, such as nonlinear shear deformations, occurring in the regions of the layers adjacent to the delaminating interface. Therefore, all the sliding phenomena are here concentrated at the interface level.

\begin{tabular}{ccccc}
\hline $\begin{array}{c}G_{\text {IIc }} \\
(\mathrm{N} / \mathrm{mm})\end{array}$ & $\begin{array}{c}\bar{\tau} \\
(\mathrm{MPa})\end{array}$ & $\begin{array}{c}\delta_{\mathrm{y}} \\
(\mathrm{mm})\end{array}$ & $\begin{array}{c}\delta_{\mathrm{f}}\left(=4 \delta_{\mathrm{y}}\right) \\
(\mathrm{mm})\end{array}$ & $\begin{array}{c}\bar{\delta}\left(=6 \delta_{\mathrm{y}}\right) \\
(\mathrm{mm})\end{array}$ \\
\hline 1.0 & 67.0 & 0.00331 & 0.01324 & 0.01986 \\
\hline
\end{tabular}

Table 4: Interface mechanical properties according to Fig. 5b.

Such basic layers are combined to get the hybrid layups in Tab. 5, each of them corresponding to a different failure mechanism.

\begin{tabular}{ccccccc}
\hline layups & $\begin{array}{c}h_{1} \\
(\mathrm{~mm})\end{array}$ & $\begin{array}{c}h_{2} \\
(\mathrm{~mm})\end{array}$ & $\begin{array}{c}\bar{\sigma}_{2} \\
(\mathrm{MPa})\end{array}$ & $\begin{array}{c}\bar{\varepsilon}_{2} \\
(\%)\end{array}$ & $\begin{array}{c}\text { mechanical } \\
\text { response }\end{array}$ \\
\hline $\mathrm{EG}_{1} / \mathrm{C}_{1} / \mathrm{EG}_{1}$ & 0.144 & 0.015 & $2339(\overline{\mathrm{w}}=53.8)$ & 2.29 & $(\mathcal{P})$ & Fig. 6 \\
$\mathrm{SG}_{1} / \mathrm{C}_{3} / \mathrm{SG}_{1}$ & 0.155 & 0.045 & $1962(\overline{\mathrm{w}}=37.9)$ & 1.93 & $(\mathcal{C})$ & Fig. 7 \\
$\mathrm{SG}_{1} / \mathrm{C}_{1} / \mathrm{SG}_{1}$ & 0.155 & 0.015 & $2339(\overline{\mathrm{w}}=44.7)$ & 2.29 & $(\mathcal{F})$ & Fig. 8 \\
$\mathrm{SG}_{1} / \mathrm{C}_{2} / \mathrm{SG}_{1}$ & 0.155 & 0.030 & $2132(\overline{\mathrm{w}}=53.8)$ & 2.10 & $(\mathcal{D})$ & Fig. 9 \\
\hline
\end{tabular}

Table 5: Considered layups. The thicknesses refer to half laminate, according to the configuration in Fig. 2.

The numerical results are compared against the experimental evidences of four tensile hybrid specimens, extracted from $[25,45]$. These specimens have been tested with a displacement controlled universal hydraulic test machine with a slow elongation rate $(2 \mathrm{~mm} / \mathrm{min})$. The freelength and width of a single specimen are, respectively, of $160 \mathrm{~mm}$ and $20 \mathrm{~mm}$, whereas the thickness changes accordingly to the different layups. Numerically, a shorter length has been considered, ten times smaller of the experimental one $(16 \mathrm{~mm})$. Such smaller length does not affect neither the failure mechanism of the laminate nor, significantly, the global response in terms of the average stress and mean strain, but facilitates the graphic representation of the failure mechanisms. Indeed, different specimen lengths only affect the stress drops amplitudes of consecutive fractures in the low-strain layer during the fragmentation process.

The numerical results are also compared against the simplified analytical model, developed in [45], based on a perfectly plastic-brittle interfacial slip law. Such analytical model, as shown by [4], is not able to characterise correctly the crack spacing of a fragmentation process. Nev- 
ertheless, it is an effective and fast tool to identify the failure mode and to get qualitatively the global response of the laminate.

Hereafter, the four numerical examples of Tab. 5 are presented, following the order of the table, and their mechanical response discussed. Each response, corresponding to a different layup, owns a linear elastic stage. The elastic stress limit, at which the first fracture in the low-strain layer occurs, is given by (5.1), whereas the apparent elastic tensile modulus reads

$$
\mathrm{E}_{\mathrm{c}}:=\frac{h_{1} \mathrm{E}_{1}+h_{2} \mathrm{E}_{2}}{h_{1}+h_{2}}
$$

As clearly shown by the results, each numerical response well predicts the global stress-strain response, the crack patters and the delamination process observed in the experiments.

The first considered layup, $\mathrm{EG}_{1} / \mathrm{C}_{1} / \mathrm{EG}_{1}$, has shown a brittle response with a premature failure $(\mathcal{P})$, Fig. 6a. Therein, significant instants, identified by circled numbers, are represented nearby in Fig. 6b, where the complete fracture of the composite is highlighted. In addition, Figures $6 \mathrm{c}$ and $6 \mathrm{~d}$ show, respectively, the stress profiles at the incipient failure, where $\alpha_{1}=\alpha_{2}=$ $\delta=0$, and the final damage profiles at failure, where $\sigma_{1}=\sigma_{2}=\tau=0$. The role of the interface, although not evident in the figures, is crucial in transferring the stress carried by the low-strain layer before the fracture to the high-strain layer. The resulting "over" stress causes the failure of the high-strain layer in the very same point and the coincident instant of the low-strain layer crack leading to the failure of the entire composite.

As pointed out in [45], E-glass based hybrid laminates, considered in [25], were not strong enough and the final failure was not significantly higher than the damage initiation point, without achieving a remarkable pseudo-ductile behaviour. Therefore, E-glass/carbon based laminates were abandoned in favour of S-glass/carbon laminates in the successive experimental campaign, [45], to improve the mechanical response.

An example of catastrophic delamination is offered by $\mathrm{SG}_{1} / \mathrm{C}_{3} / \mathrm{SG}_{1}$ layup. The typical features of such failure mechanism are clearly deduced from the stress-strain response of Fig. $7 \mathrm{a}$ with the salient response snapshots highlighted in Fig. 7b. Once the elastic stress limit in the low-strain layer has been reached, a large delamination of the interface instantaneously appears. Consequently, the overall stress significantly decreases and further load increments extend the delaminated region at approximately constant stress. Once the delamination fronts have almost reached the bar ends, the stress starts smoothly to increase again with the same elastic modulus of the high-strain layer, until the very same layer fails. The stress and damage profiles immediately after the failure of the low-strain material are reported in Fig. 7c and Fig. 7d, respectively. Therein it is worth noting the finite length interface stress transfer region, corresponding to non-vanishing $\tau$ points. The damage profiles after the complete failure of the laminate are shown in Fig. 7e. Clearly, in such a case, the fracture of the high-strain material occurs in an arbitrary point of the delaminated region, not coinciding with the location of the low-strain fracture, according to the Weibull's statistical distribution (5.2).

To obtain fragmentation $(\mathcal{F})$ in the low-strain layer, a thinner high-strain layer must be used, as in the $\mathrm{SG}_{1} / \mathrm{C}_{1} / \mathrm{SG}_{1}$ layup. The global stress-strain response in Fig. 8a and the respective fracture patterns in Fig. 8b permit to unveil two linear elastic regimes with in between a knurled non-linear transition regime. Each small stress drop is associated to a new crack development in the low-strain layer realising a fragmentation process. Intermediate and final fracture distributions snapshots are shown in Fig. 8c and Fig. 8e, respectively. As highlighted by the interface state, Fig. $8 \mathrm{~b}$ and Fig. 8 d, the slip never overcomes the critical value $\bar{\delta}$, meaning that delamination in the interface never occurs, due to the too small thickness of the low-strain layer. Correspondingly, the fracture of the high-strain material occurs at the same position of 


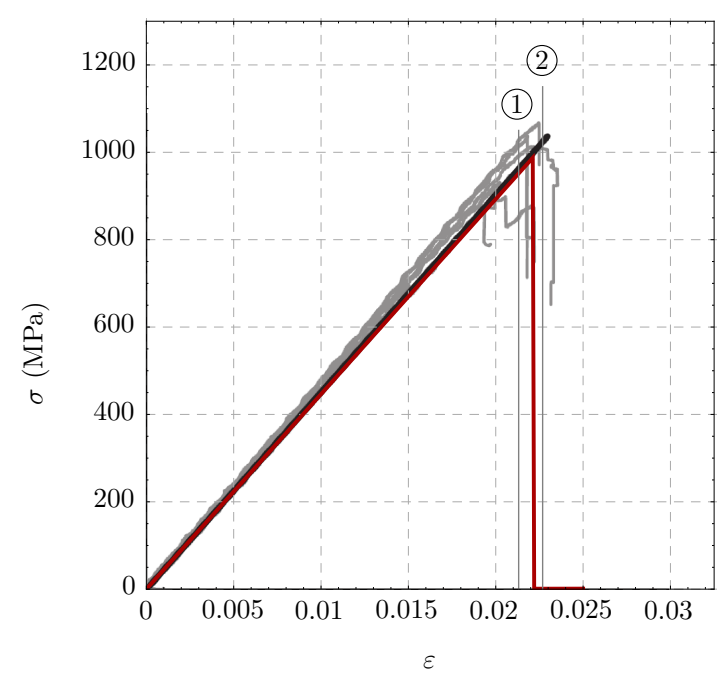

(a)
(1)

(2)

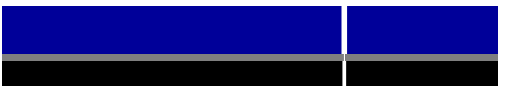

(b)

(1)

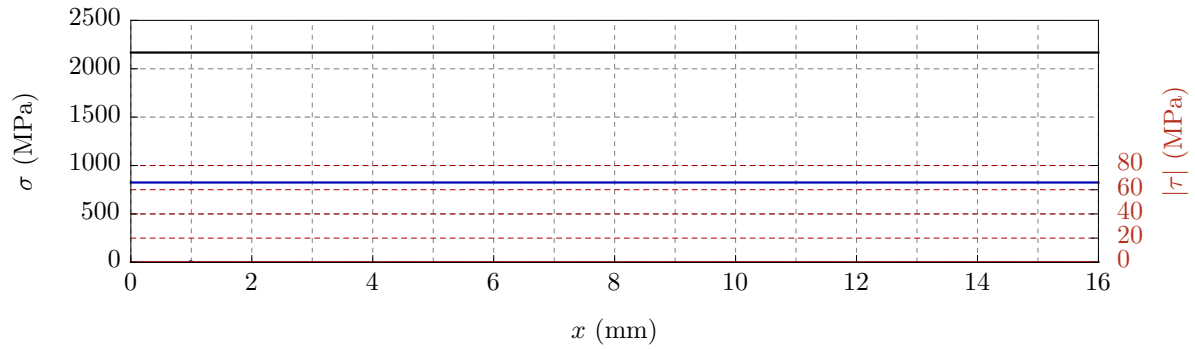

(c)

(2)

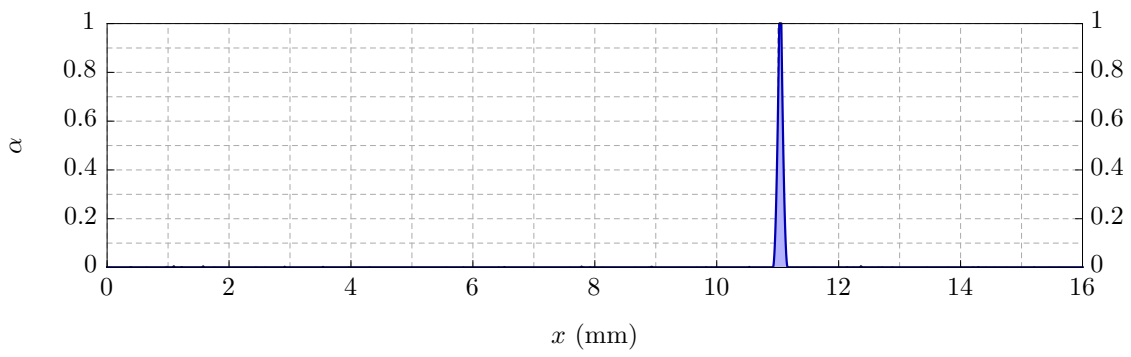

(d)

Figure 6: Numerical results for the $\mathrm{EG}_{1} / \mathrm{C}_{1} / \mathrm{EG}_{1}$ model: (a) Global stress-strain response. The black and gray curves represent, respectively, the analytical and experimental results in [45], whereas the red curve represents the numerical response; (b) Mechanical response snapshots. The blue, black and gray regions represent, respectively, the unbroken high-strain layer, the unbroken low-strain layer and the unstressed interface. White lines denote the occurrence of a fracture; (c) Stress profiles at the instant identified by the circled number. Blue, black and red curves represent, respectively, $\sigma_{1}, \sigma_{2}$ and $\tau$, this last always vanishing for the present case; (d) Damage profiles at the instant identified by the circled number. Filled blue and black curves represent, respectively, $\alpha_{1}, \alpha_{2}$, in this case overlapping.

a previous fracture in the low-strain layer.

The last layup $\mathrm{SG}_{1} / \mathrm{C}_{2} / \mathrm{SG}_{1}$, with an intermediate low-strain layer thickness with respect to the two former ones, has been able to describe a fragmentation with a diffuse delamination $(\mathcal{D})$ failure process. After reaching the elastic limit, a fragmentation process in the low-strain layer starts, very similar to the previous response. Similar knurls are observed in the stress-strain diagram in Fig. 9a. At a certain load level, the fragmentation process stops in favor of a delamination process. Indeed, delamination fronts start to propagate around the cracks of the 
low-strain layer, Fig. 9b. It is worth to remark that the delamination process always follows fragmentation, as rigorously explained in [4], in the sense that once the interface between two consecutive cracks of the low-strain layer starts to delaminate, the stress of the low-strain layer in the very same region reduces and the critical stress cannot be attained anymore. Instead, during such a stage, the stress in the high-strain layer increases, causing at a certain point its failure in some delaminated points, and therefore the failure of the entire composite. Intermediate damage and stress profiles are represented in Fig. 9c and Fig. 9d, respectively, whereas the final damage profiles is visualised in Fig. 9e.

The dispersed fragmentation and delamination in this layup lead to a final failure strain significantly higher than the carbon failure strain. It is worth to point out that the small load drops in the response tend to decrease (and in the limit to vanish) if longer bars are considered. For such a response, the value of the pseudo-ductile strain, that is the difference between the ultimate strain and the elastic strain limit, is approximately equal to $1.2 \%$, corresponding to an increment of approximately $50 \%$ of final failure strain. Moreover, this high pseudo-ductile response has been achieved with a load increment ranging from $1150 \mathrm{MPa}$ (elastic limit) up to $1350 \mathrm{MPa}$ (failure) $(\Delta \sigma=+200 \mathrm{MPa} /+17 \%)$.

All the global stress-strain curves are in very good agreement with the experimental results.

Figs. 10a-10c show the energy diagrams of the S-based hybrid layups. Such energetic analyses allow, for instance, to highlight how energy is distributed during the failure process or to evaluate the dissipative capabilities of the different failure modes. Indeed, one may clearly observe in Fig. 10a the energy distribution during the fragmentation process, where part of the elastic potential energy is dissipated in creating fractures. In addition, the dissipation of the pseudoductile layup $\left(\mathrm{SG}_{1} / \mathrm{C}_{2} / \mathrm{SG}_{1}\right)$, Fig. 10c, is caused first by the fragmentation process and then by a diffuse delamination. The resulting total dissipated energy is just slightly lower than the energy dissipated by the catastrophic delamination mode $\left(\mathrm{SG}_{1} / \mathrm{C}_{3} / \mathrm{SG}_{1}\right)$, which, for the present layups, is the most dissipative mode, but at the price of a thicker configuration and a deep unwanted load drop at the end of the elastic stage, Fig. 10b.

Concerning the interface, the failure mode strongly depends on the maximum allowable shear stress $\bar{\tau}$ and the fracture toughness $G_{\mathrm{II} c}$. Instead, the kind of interface law, given $\bar{\tau}$ and $G_{\text {IIc }}$, has an impact only on the cracks spacing during the fragmentation process. The reader can find further details about the sensitivity of the failure modes with respect to the interface law and other constitutive and geometrical parameters in [4]. 


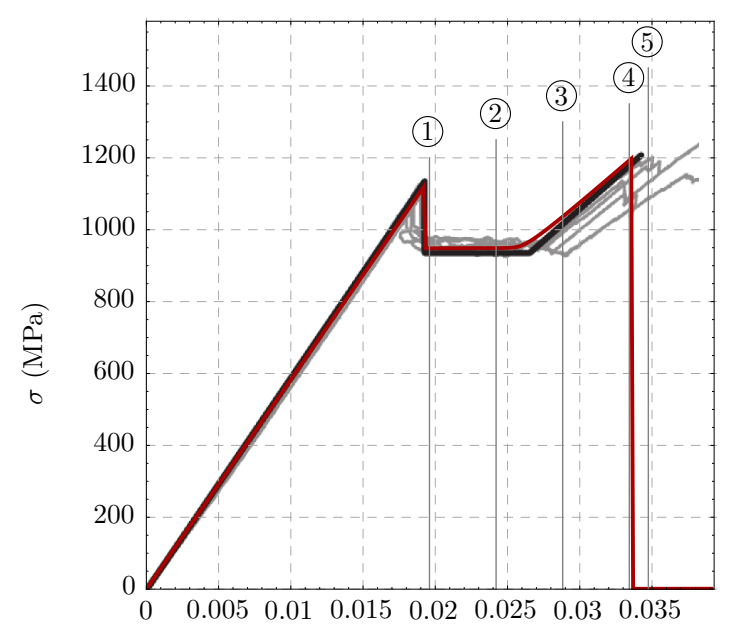

$\varepsilon$
(1)

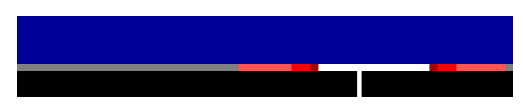

(2)

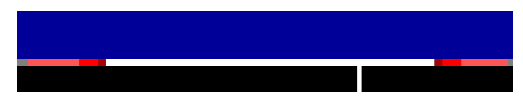

(3)

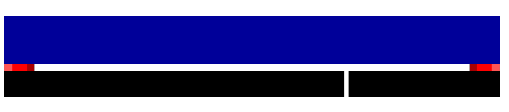

(4)

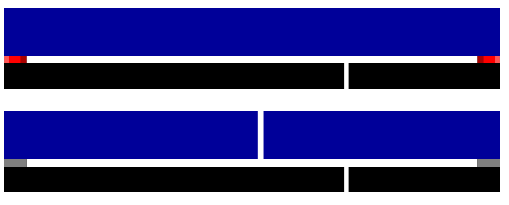

(b)

(a)

(1)

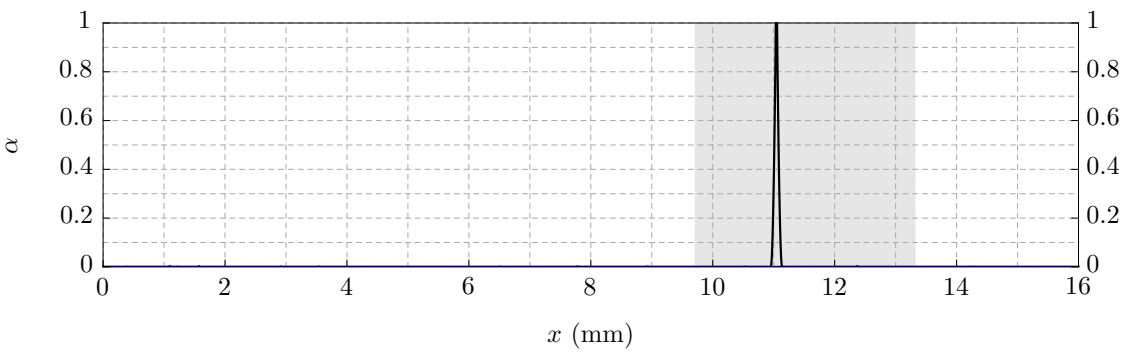

(c)

(1)

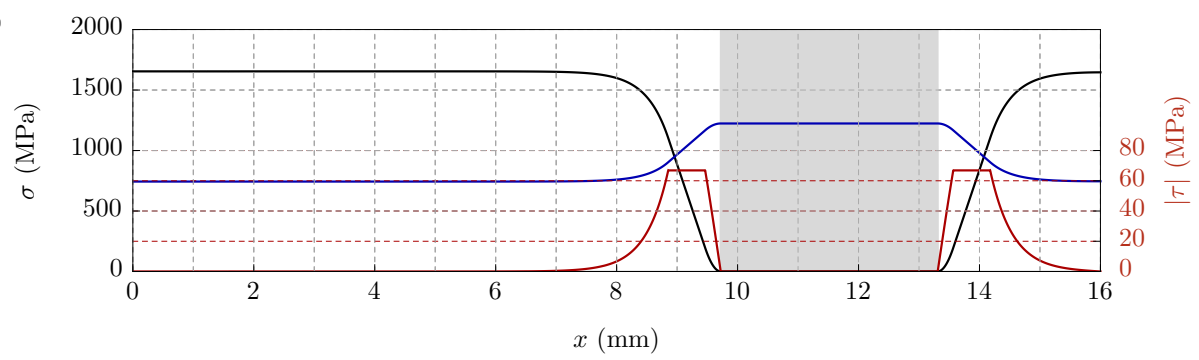

(d)

(6)

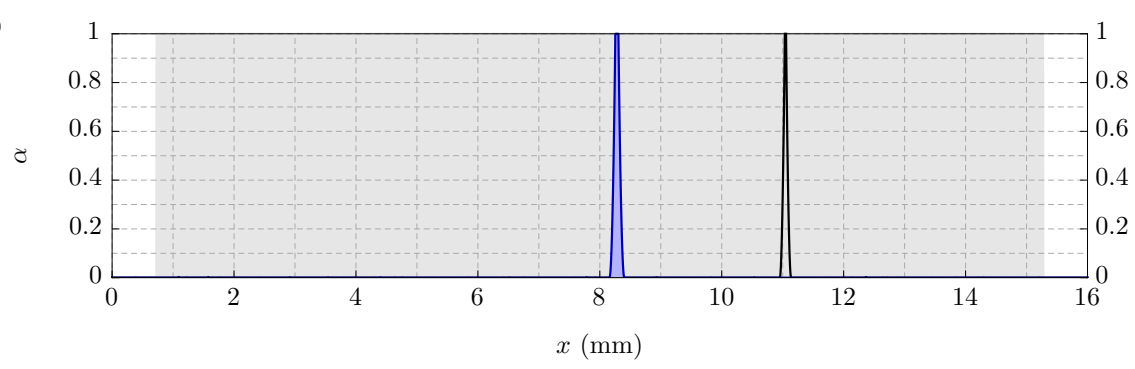

(e)

Figure 7: Numerical results for the $\mathrm{SG}_{1} / \mathrm{C}_{3} / \mathrm{SG}_{1}$ model: (a) Global stress-strain response. The black and gray curves represent, respectively, the analytical and experimental results in [45], whereas the red curve represents the numerical response; (b) Mechanical response snapshots. The blue, black and gray regions represent, respectively, the unbroken high-strain layer, the unbroken low-strain layer and the unstressed interface. White lines denote the occurrence of a fracture or delamination. Red colours identify the interface loading state: light, neutral and dark red, for, respectively, the hardening, constant and softening stage; (c) and (e) Damage profiles at the instant identified by the circled number. Filled blue and black curves represent, respectively, $\alpha_{1}, \alpha_{2}$. The gray region identifies the delaminated interface zone; (d) Stress profiles at the instant identified by the circled number. Blue, black and red curves represent, respectively, $\sigma_{1}, \sigma_{2}$ and $\tau$. 


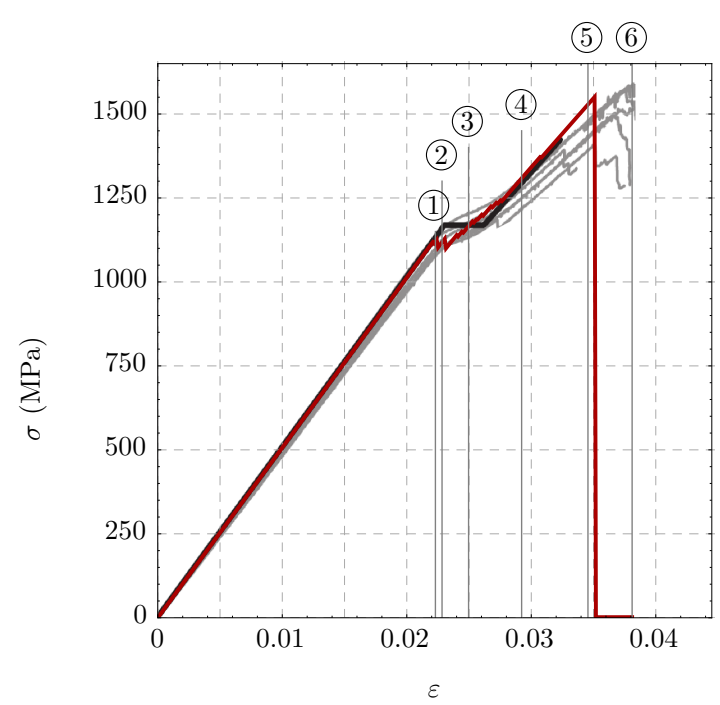

(a)
(1)

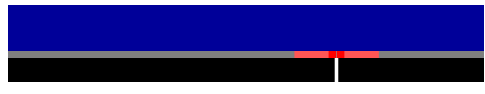

(2)

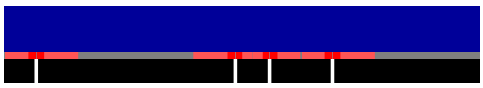

(3)

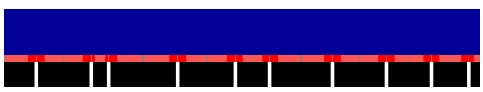

(4)

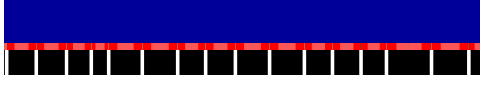

(5)

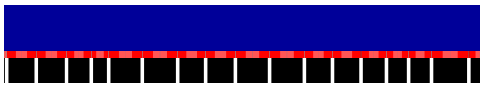

(6)

(3)

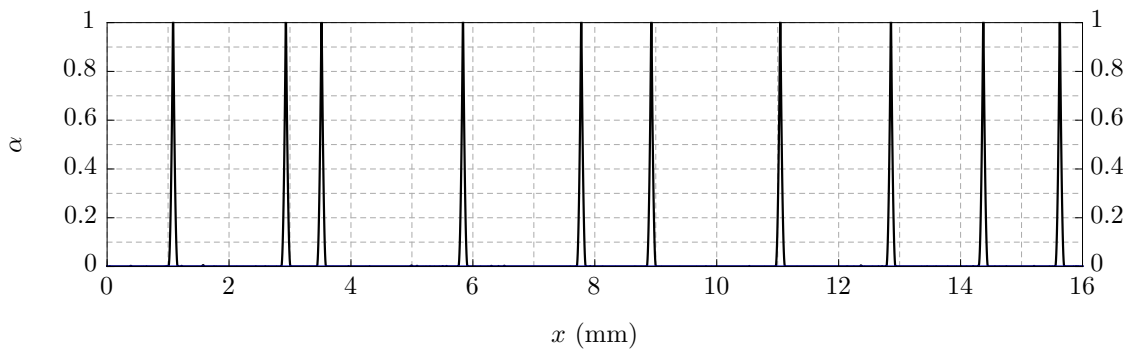

(c)

(3)

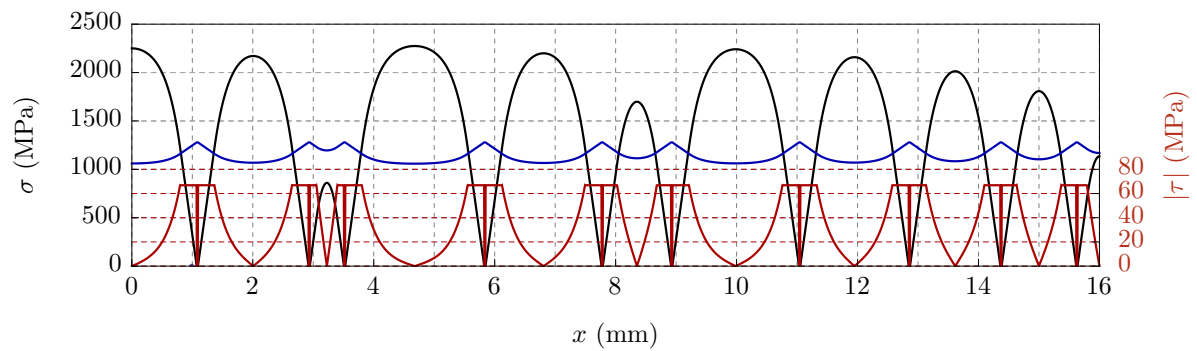

(d)

(6)

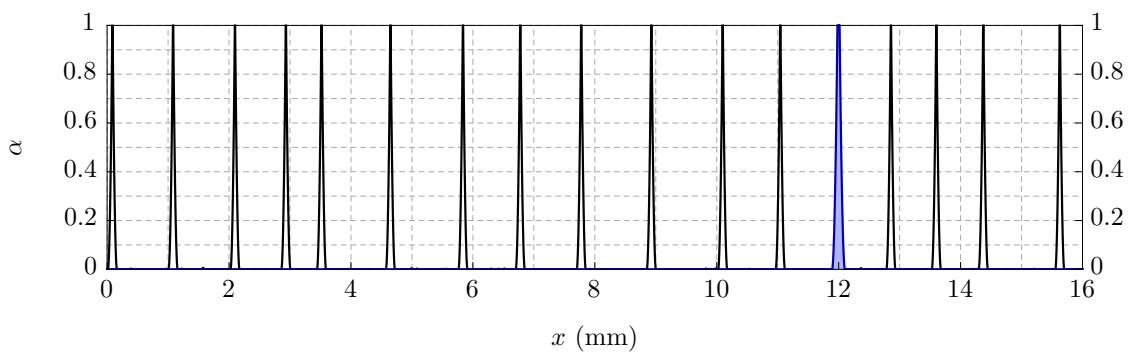

(e)

Figure 8: Numerical results for the $\mathrm{SG}_{1} / \mathrm{C}_{1} / \mathrm{SG}_{1}$ model: (a) Global stress-strain response. The black and gray curves represent, respectively, the analytical and experimental results in [45], whereas the red curve represents the numerical response; (b) Mechanical response snapshots. The blue, black and gray regions represent, respectively, the unbroken high-strain layer, the unbroken low-strain layer and the unstressed interface. White lines denote the occurrence of a fracture or delamination. Red colours identify the interface loading state: light, neutral and dark red, for, respectively, the hardening, constant and softening stage; (c) and (e) Damage profiles at the instant identified by the circled number. Filled blue and black curves represent, respectively, $\alpha_{1}, \alpha_{2}$; (d) Stress profiles at the instant identified by the circled number. Blue, black and red curves represent, respectively, $\sigma_{1}, \sigma_{2}$ and $\tau$. 


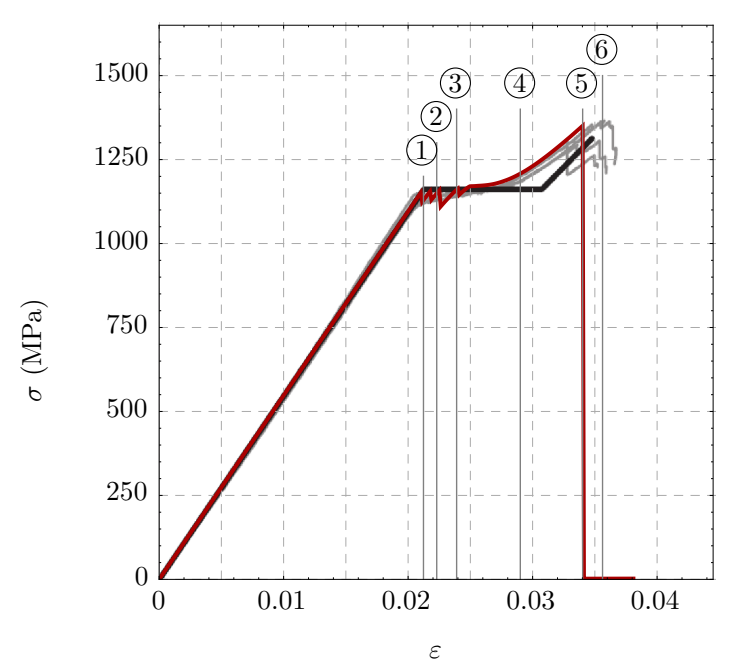

(a)
(1)

(2)

(3)

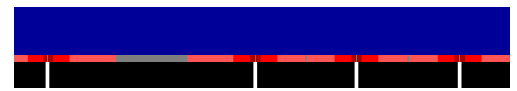

(4)

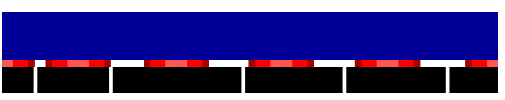

(5)

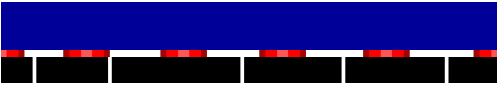

(6)

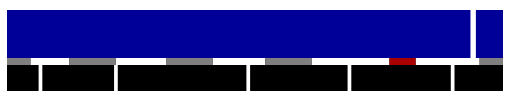

(b)

(4)

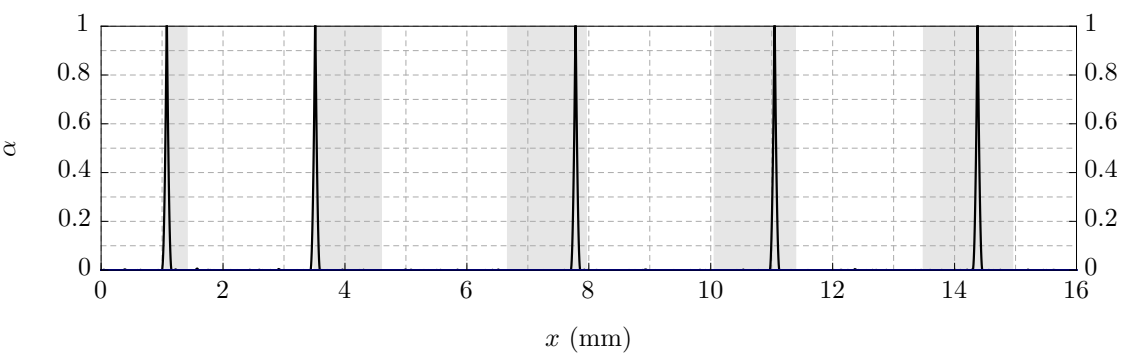

(c)

(4)

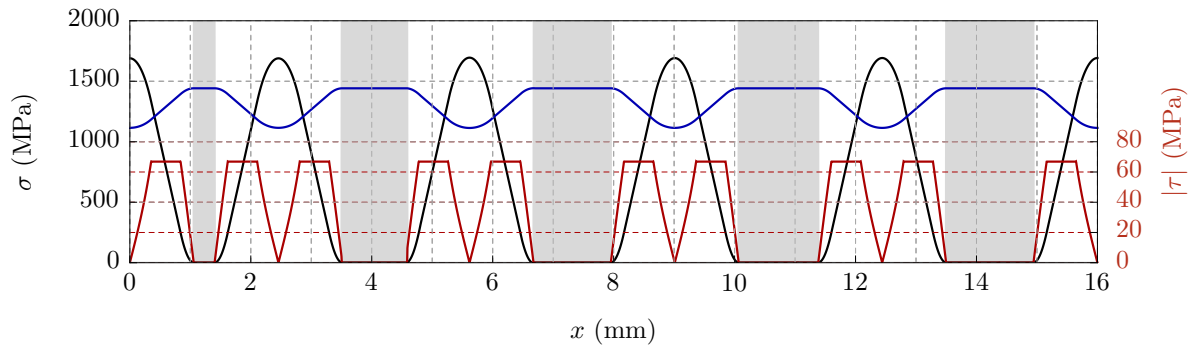

(d)

(6)

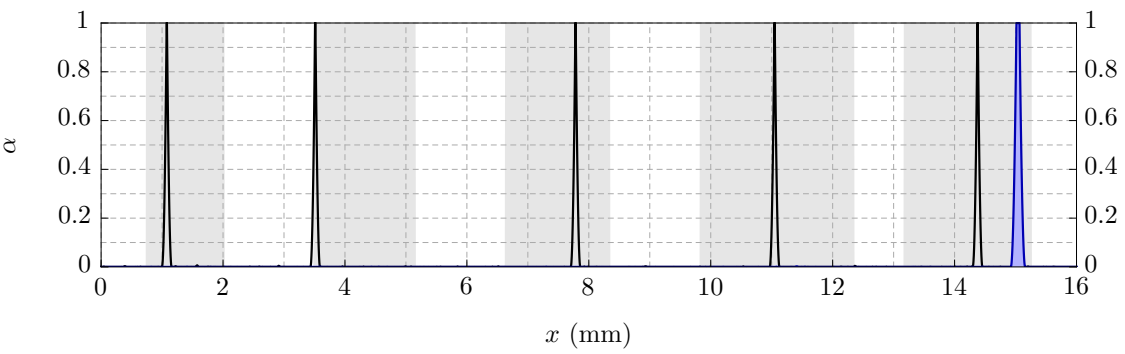

(e)

Figure 9: Numerical results for the $\mathrm{SG}_{1} / \mathrm{C}_{2} / \mathrm{SG}_{1}$ model: (a) Global stress-strain response. The black and gray curves represent, respectively, the analytical and experimental results in [45], whereas the red curve represents the numerical response; (b) Mechanical response snapshots. The blue, black and gray regions represent, respectively, the unbroken high-strain layer, the unbroken low-strain layer and the unstressed interface. White lines denote the occurrence of a fracture or delamination. Red colours identify the interface loading state: light, neutral and dark red, for, respectively, the hardening, constant and softening stage; (c) and (e) Damage profiles at the instant identified by the circled number. Filled blue and black curves represent, respectively, $\alpha_{1}, \alpha_{2}$. The gray regions identify the delaminated interface zones; (d) Stress profiles at the instant identified by the circled number. Blue, black and red curves represent, respectively, $\sigma_{1}, \sigma_{2}$ and $\tau$. 

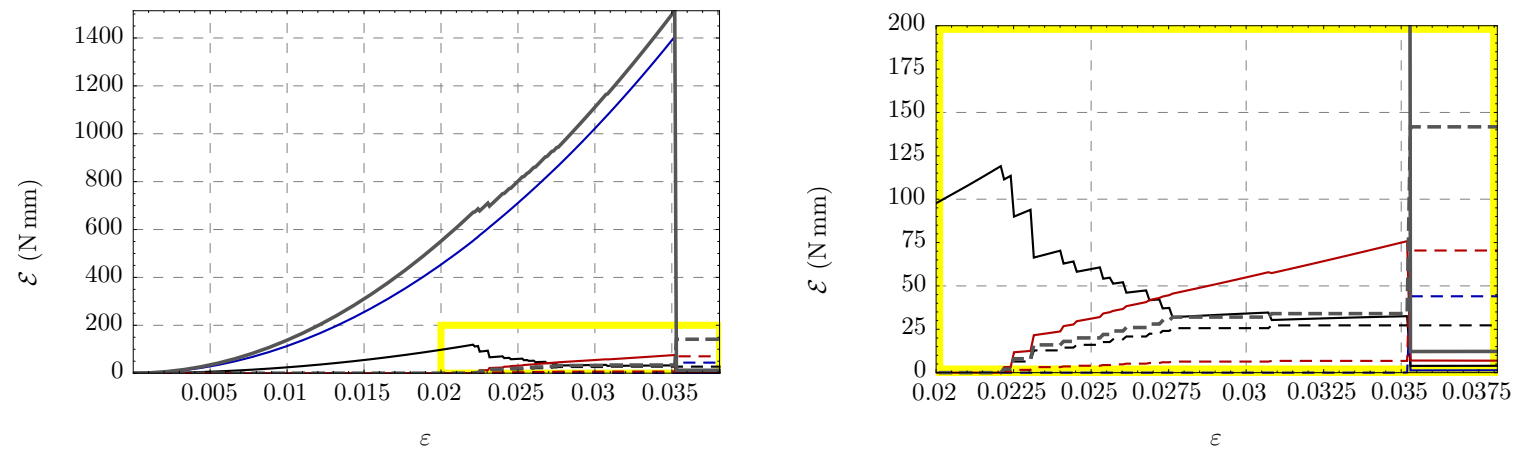

(a) $\mathrm{SG}_{1} / \mathrm{C}_{1} / \mathrm{SG}_{1}$
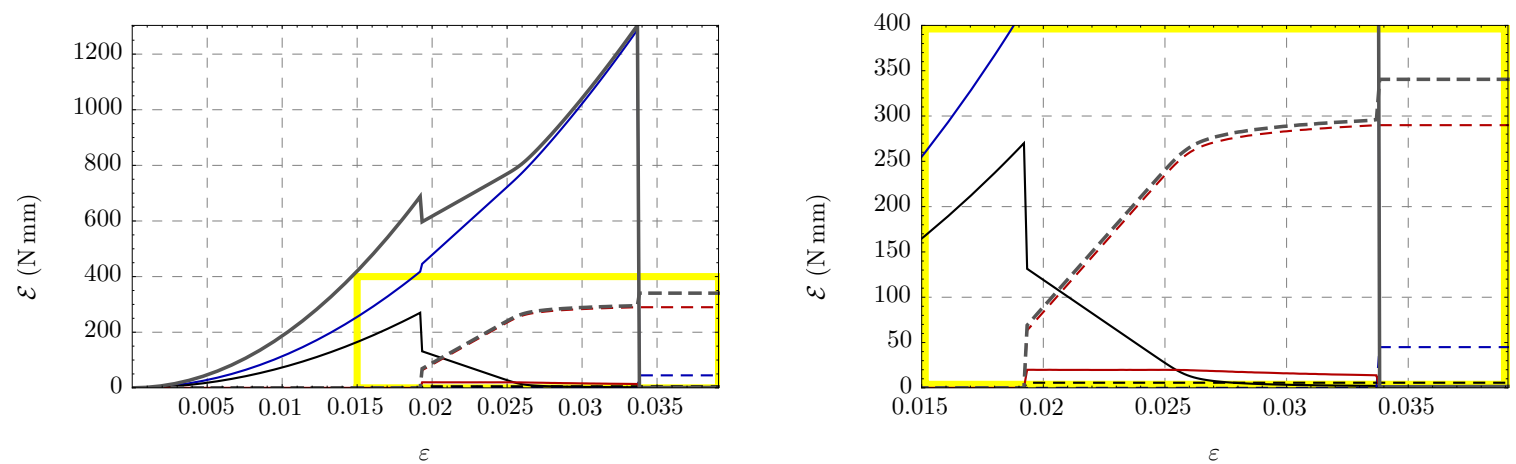

(b) $\mathrm{SG}_{1} / \mathrm{C}_{3} / \mathrm{SG}_{1}$
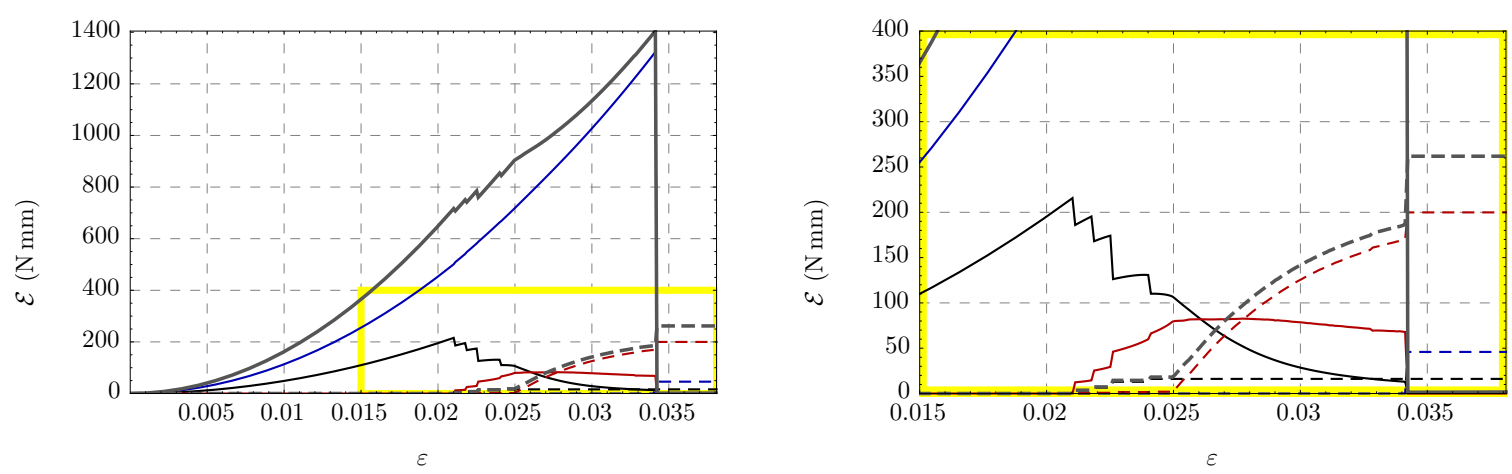

(c) $\mathrm{SG}_{1} / \mathrm{C}_{2} / \mathrm{SG}_{1}$

Figure 10: Energetic diagrams. Continuous lines represent potential energy contributions whereas dashed lines refer to dissipation. Gray curves correspond to total quantities, blue curves to $\Omega_{1}$ quantities, black curves to $\Omega_{2}$ quantities and red curves to the interface contributions. The yellow box on the left pictures identify the regions magnified on the right pictures 


\section{Conclusion and perspectives}

Ductility is a fundamental requirement for composite materials of the future. In fact, more effective mechanical behaviours will expand the already vast range of applications of these materials. This feature is obtained at the price of a much more complex failure mechanism that requires specific design criteria and calibrated procedures in order to optimise the structural response of the elements.

For this purpose, it is necessary not only to experimentally understand the different dissipative mechanisms that characterise the rupture, but also to formulate a consistent model, in order to develop accurate and reliable numerical tools for the design of the composite.

The proposed phase-field modeling strategy permits, even in a simplified 1D setup, to completely describe the four different failure mechanisms of hybrid laminates that are usually reported in the DMM schematisation. In particular, with such approach, cracks distribution and density within each material can be accurately determined, without the need to introduce predefined crack loci. Moreover, the delamination mechanism has been fully exploited. Indeed, the energetic variational investigation has permitted to determine the dissipative contribution of the different failure mechanisms. Moreover, crack patterns densities, which are an intrinsic characteristic of the hybrid composite, [4], could be of help in determining the damage state of the structural element in order to meet damage tolerance requirements.

This work represents a first attempt to numerically describe the mechanical response of hybrid laminates. At the present stage, two natural extensions are possible. Firstly, in a full 2D simulation in plane regime it would be possible to capture the sliding mechanisms that are not limited at the interface level but also interest the material [30] and capture the bending effects outlined in [43]. Alternatively, the present formulation could be extended to a $2 \mathrm{D}$ formulation in membranal state of stress. This enrichment would permit to describe the delamination that is not a simple 1D process as illustrated in [25,38]. Moreover, in 2D configurations non uniform boundary conditions or material anysotropies could be introduced to highlight the occurrence of complicated and irregular crack patterns.

\section{Acknowledgements}

R. Alessi wishes to acknowledge the financial support of the MATHTECH-CNR-INdAM project.

\section{Appendix A. Construction of the evolution equations from the energy balance and the stability condition}

In this section, we explicitly show how to derive the evolution laws reported in Tab. 2. By considering the first-order stability condition (st) and using the definitions of the total internal potential energy (3.8) and of the total dissipated work (3.13), we get

$$
\begin{aligned}
0 \leq & \mathcal{E}^{\prime}\left(u_{1}, \alpha_{1}, u_{2}, \alpha_{2}, \delta_{\mathrm{c}}\right)\left(\widetilde{u}_{1}, \widetilde{\alpha}_{1}, \widetilde{u}_{2}, \widetilde{\alpha}_{2}, 0\right)+\mathcal{D}^{\prime}\left(\alpha_{1}, \alpha_{2}, \delta_{\mathrm{c}}\right)\left(\widetilde{\alpha}_{1}, \widetilde{\alpha}_{2}, 0\right) \\
\leq & \int_{0}^{L} h_{1} \sigma_{1} \widetilde{u}_{1}^{\prime}+\tau \widetilde{u}_{1} \mathrm{~d} x+\int_{0}^{L} h_{2} \sigma_{2} \widetilde{u}_{2}^{\prime}-\tau \widetilde{u}_{2} \mathrm{~d} x+ \\
& +\int_{0}^{L} h_{1}\left(-\frac{1}{2} \mathrm{~S}_{1}^{\prime}\left(\alpha_{1}\right) \sigma_{1}^{2}+\mathrm{w}_{1}^{\prime}\left(\alpha_{1}\right)\right) \widetilde{\alpha}_{1}+h_{1} \overline{\mathrm{w}}_{1} \eta_{1}^{2} \alpha_{1}^{\prime} \widetilde{\alpha}_{1}^{\prime} \mathrm{d} x+ \\
& +\int_{0}^{L} h_{2}\left(-\frac{1}{2} \mathrm{~S}_{2}^{\prime}\left(\alpha_{2}\right) \sigma_{2}^{2}+\mathrm{w}_{2}^{\prime}\left(\alpha_{2}\right)\right) \widetilde{\alpha}_{2}+h_{2} \overline{\mathrm{w}}_{2} \eta_{2}^{2} \alpha_{2}^{\prime} \widetilde{\alpha}_{2}^{\prime} \mathrm{d} x
\end{aligned}
$$


where the dependence of each variable to the spatial coordinate has been dropped and where the stress notions (3.7) have been used. Since the inequality (A.1) must hold for any test direction $\left(\widetilde{u}_{1}, \widetilde{u}_{2}, \widetilde{\alpha}_{1}, \widetilde{\alpha}_{2}\right)$ such that $\widetilde{u}_{i}(0)=\widetilde{u}_{i}(L)=0$ and $\widetilde{\alpha}_{i} \geq 0$ (with $\widetilde{\alpha}_{i}=0$ where $\alpha_{i}=1$ ), we easily derive the following results:

(i) Equilibrium equations for $\Omega_{1}$ and $\Omega_{2}$. Taking first $\widetilde{u}_{1} \neq 0$ and all other test directions equal to zero, we get

$$
\begin{aligned}
0 & \leq \int_{0}^{L} h_{1} \sigma_{1} \widetilde{u}_{1}^{\prime}+\tau \widetilde{u}_{1} \mathrm{~d} x= \\
& =\int_{0}^{L}\left(-h_{1} \sigma_{1}^{\prime}+\tau\right) \widetilde{u}_{1} \mathrm{~d} x
\end{aligned}
$$

which holds if and only if

$$
h_{1} \sigma_{1}^{\prime}-\tau=0
$$

where the integration by parts has been used. Similarly, taking $\widetilde{u}_{2} \neq 0$ and all other test directions equal to zero, we get

$$
h_{2} \sigma_{2}^{\prime}+\tau=0
$$

(ii) Damage inequalities for $\Omega_{1}$ and $\Omega_{2}$. Taking now $\widetilde{\alpha}_{1} \neq 0$ and all other test directions equal to zero, we get

$$
\begin{aligned}
0 & \leq \int_{0}^{L} h_{1}\left(-\frac{1}{2} \mathrm{~S}_{1}^{\prime}\left(\alpha_{1}\right) \sigma_{1}^{2}+\mathrm{w}_{1}^{\prime}\left(\alpha_{1}\right)\right) \widetilde{\alpha}_{1}+h_{1} \overline{\mathrm{w}}_{1} \eta_{1}^{2} \alpha_{1}^{\prime} \widetilde{\alpha}_{1}^{\prime} \mathrm{d} x \\
& =\int_{0}^{L} h_{1}\left(-\frac{1}{2} \mathrm{~S}_{1}^{\prime}\left(\alpha_{1}\right) \sigma_{1}^{2}+\mathrm{w}_{1}^{\prime}\left(\alpha_{1}\right)-\overline{\mathrm{w}}_{1} \eta_{1}^{2} \alpha_{1}^{\prime \prime}\right) \widetilde{\alpha}_{1} \mathrm{~d} x+\left[\alpha_{1}^{\prime} \widetilde{\alpha}_{1}\right]_{0}^{L}
\end{aligned}
$$

which holds if and only if

$$
\underbrace{\frac{1}{2} \mathrm{~S}_{1}^{\prime}\left(\alpha_{1}\right) \sigma_{1}^{2}-\mathrm{w}_{1}^{\prime}\left(\alpha_{1}\right)+\overline{\mathrm{w}}_{1} \eta_{1}^{2} \alpha_{1}^{\prime \prime}}_{f_{\mathrm{d}_{1}}\left(\sigma_{1}, \alpha_{1}\right)} \leq 0,
$$

and

$$
\alpha_{1}^{\prime}(L)=-\alpha_{1}^{\prime}(0) \geq 0
$$

where again the integration by parts for the damage variable has been used. Similarly, taking $\widetilde{\alpha}_{2} \neq 0$ and all other test directions equal to zero, we get

$$
\underbrace{\frac{1}{2} \mathrm{~S}_{2}^{\prime}\left(\alpha_{2}\right) \sigma_{2}^{2}-\mathrm{w}_{2}^{\prime}\left(\alpha_{2}\right)+\overline{\mathrm{w}}_{2} \eta_{2}^{2} \alpha_{2}^{\prime \prime}}_{f_{\mathrm{d} 2}\left(\sigma_{2}, \alpha_{2}\right)} \leq 0,
$$

and

$$
\alpha_{2}^{\prime}(L)=-\alpha_{2}^{\prime}(0) \geq 0
$$

Now, by considering the first order energy balance (eb) and using the definitions of the total internal potential energy (3.8) and of the total dissipated work (3.13) together with the 
boundary conditions (3.1), we get, taking again advantage of the integration by parts,

$$
\begin{aligned}
& 0=\frac{\mathrm{d}}{\mathrm{d} t}\left(\mathcal{E}\left(u_{1}, \alpha_{1}, u_{2}, \alpha_{2}, \delta_{\mathrm{c}}\right)+\mathcal{D}\left(\alpha_{1}, \alpha_{2}, \delta_{\mathrm{c}}\right)-\mathcal{L}(t)\right) \\
& =\int_{0}^{L} h_{1} \sigma_{1} \dot{u}_{1}^{\prime}+\tau \dot{u}_{1} \mathrm{~d} x+\left(h_{1} \sigma_{1}(L)-f_{1}\right) \dot{\bar{u}}+ \\
& +\int_{0}^{L} h_{2} \sigma_{2} \dot{u}_{2}^{\prime}+\tau \dot{u}_{2} \mathrm{~d} x+\left(h_{2} \sigma_{2}(L)-f_{2}\right) \dot{\bar{u}}+ \\
& +\int_{0}^{L} h_{1}\left(-\frac{1}{2} \mathrm{~S}_{1}^{\prime}\left(\alpha_{1}\right) \sigma_{1}^{2}+\mathrm{w}_{1}^{\prime}\left(\alpha_{1}\right)\right) \dot{\alpha}_{1}+h_{1} \overline{\mathrm{w}}_{1} \eta_{1}^{2} \alpha_{1}^{\prime} \dot{\alpha}_{1}^{\prime} \mathrm{d} x+ \\
& +\int_{0}^{L} h_{2}\left(-\frac{1}{2} \mathrm{~S}_{2}^{\prime}\left(\alpha_{2}\right) \sigma_{2}^{2}+\mathrm{w}_{2}^{\prime}\left(\alpha_{2}\right)\right) \dot{\alpha}_{2}+h_{2} \overline{\mathrm{w}}_{2} \eta_{2}^{2} \alpha_{2}^{\prime} \dot{\alpha}_{2}^{\prime} \mathrm{d} x
\end{aligned}
$$

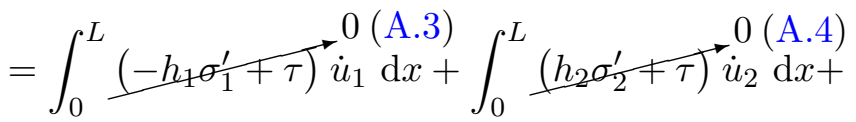

$$
\begin{aligned}
& +\left(h_{1} \sigma_{1}(L)-f_{1}\right) \dot{\bar{u}}+\left(h_{2} \sigma_{2}(L)-f_{2}\right) \dot{\bar{u}}+ \\
& +\int_{0}^{L} h_{1} \underbrace{\left(-\frac{1}{2} \mathrm{~S}_{1}^{\prime}\left(\alpha_{1}\right) \sigma_{1}^{2}+\mathrm{w}_{1}^{\prime}\left(\alpha_{1}\right)-\overline{\mathrm{w}}_{1} \eta_{1}^{2} \alpha_{1}^{\prime \prime}\right)}_{=-f_{\mathrm{d} 1}\left(\sigma_{1}, \alpha_{1}\right)} \dot{\alpha}_{1} \mathrm{~d} x+\left[\alpha_{1}^{\prime} \dot{\alpha}_{1}\right]_{0}^{L} \\
& +\int_{0}^{L} h_{2} \underbrace{\left(-\frac{1}{2} \mathrm{~S}_{2}^{\prime}\left(\alpha_{2}\right) \sigma_{2}^{2}+\mathrm{w}_{2}^{\prime}\left(\alpha_{2}\right)-\overline{\mathrm{w}}_{2} \eta_{2}^{2} \alpha_{2}^{\prime \prime}\right)}_{=-f_{\mathrm{d} 2}\left(\sigma_{2}, \alpha_{2}\right)} \dot{\alpha}_{2} \mathrm{~d} x+\left[\alpha_{2}^{\prime} \dot{\alpha}_{2}\right]_{0}^{L}
\end{aligned}
$$

where the dependence of each variable to the spatial coordinate has been dropped and where the stress notions (3.7) have been used.

In order to satisfy (A.10), we obtain, taking advantage of the first order stability results and the irreversibility conditions (ir), the following results:

(i) Equilibrium boundary conditions in $x=L$.

$$
h_{1} \sigma_{1}(L)=f_{1} \quad \text { and } \quad h_{2} \sigma_{2}(L)=f_{2}
$$

(ii) Damage consistency conditions for $\Omega_{1}$ and $\Omega_{2}$. Since $\dot{\alpha}_{1} \geq 0$ and $\dot{\alpha}_{2} \geq 0$ we get

$$
f_{\mathrm{d} 1}\left(\sigma_{1}, \alpha_{1}\right) \dot{\alpha}_{1}=0 \quad \text { and } \quad f_{\mathrm{d} 2}\left(\sigma_{2}, \alpha_{2}\right) \dot{\alpha}_{2}=0
$$

(iii) Damage boundary consistency conditions $(x=0$ and $x=L)$.

$$
\alpha_{1}^{\prime}(0) \dot{\alpha}_{1}(0)=\alpha_{1}^{\prime}(L) \dot{\alpha}_{1}(L)=\alpha_{2}^{\prime}(0) \dot{\alpha}_{2}(0)=\alpha_{2}^{\prime}(L) \dot{\alpha}_{2}(L)=0
$$

With respect to Tab. 2, (A.3), (A.4) and (A.11) furnish the bulk and boundary equilibrium equations. The conditions (A.6), (A.8) and (A.12), together with the irreversibility conditions (ir) provide the bulk damage conditions (KKT system). Moreover, from the irreversibility conditions (ir), (A.7), (A.9) and (A.13) we obtain the boundary condtions for the damage evolution, namely

$$
\alpha_{1}^{\prime}(0)=\alpha_{1}^{\prime}(L)=\alpha_{2}^{\prime}(0)=\alpha_{2}^{\prime}(L)=0
$$




\section{References}

[1] R. Abdelmoula and J.-J. Marigo, Effective behavior of a fiber bridged crack, Journal of the Mechanics and Physics of Solids, 48 (2000), pp. 2419-2444.

[2] R. ALESSI, Energetic formulation for rate-independent processes: remarks on discontinuous evolutions with a simple example, Acta Mechanica, 227 (2016), pp. 2805-2829.

[3] R. Alessi and D. Bernardini, Analysis of localization phenomena in Shape Memory Alloys bars by a variational approach, International Journal of Solids and Structures, 73-74 (2015), pp. 113-133.

[4] R. Alessi, J. Ciambella, and A. Paolone, Damage evolution and debonding in hybrid laminates with a cohesive interfacial law, Meccanica, 52 (2017), pp. 1079-1091.

[5] R. Alessi, J.-J. Marigo, C. Maurini, And S. Vidoli, Coupling damage and plasticity for a phase-field regularisation of brittle, cohesive and ductile fracture: one-dimensional examples, International Journal of Mechanical Sciences, In press (2017).

[6] R. Alessi, J.-J. Marigo, And S. Vidoli, Gradient Damage Models Coupled with Plasticity and Nucleation of Cohesive Cracks, Archive for Rational Mechanics and Analysis, 214 (2014), pp. 575-615.

[7] _ Gradient damage models coupled with plasticity: Variational formulation and main properties, Mechanics of Materials, 80 (2015), pp. 351-367.

[8] R. Alessi And K. Pham, Variational formulation and stability analysis of a three dimensional superelastic model for shape memory alloys, Journal of the Mechanics and Physics of Solids, 87 (2016), pp. 150-176.

[9] M. Ambati, T. Gerasimov, And L. De Lorenzis, A review on phase-field models of brittle fracture and a new fast hybrid formulation, Computational Mechanics, 55 (2015), pp. 383-405.

[10] L. Ambrosio and V. M. Tortorelli, On the approximation of free discontinuity problems, Boll. Un. Mat. Ital., 6-B (1992), pp. 105-123.

[11] H. Amor, J.-J. Marigo, And C. Maurini, Regularized formulation of the variational brittle fracture with unilateral contact: Numerical experiments, Journal of the Mechanics and Physics of Solids, 57 (2009), pp. 1209-1229.

[12] S. Balay, S. Abhyankar, M. Adams, J. Brown, P. Brune, K. Buschelman, L. Dalcin, V. Eijkhout, W. Gropp, D. Kaushik, M. Knepley, L. C. Mcinnes, K. Rupp, B. Smith, S. Zampini, And H. Zhang, PETSc Web page. http://www.mcs.anl.gov/petsc, 2015.

[13] J. Bleyer and J.-F. Molinari, Microbranching instability in phase-field modelling of dynamic brittle fracture, Applied Physics Letters, 110 (2017), p. 151903.

[14] J. Bleyer, C. Roux-Langlois, And J.-F. Molinari, Dynamic crack propagation with a variational phase-field model: limiting speed, crack branching and velocity-toughening mechanisms, International Journal of Fracture, 204 (2017), pp. 79-100.

[15] B. Bourdin, G. A. Francfort, and J.-J. Marigo, Numerical experiments in revisited brittle fracture, Journal of the Mechanics and Physics of Solids, 48 (2000), pp. 797-826. 
[16] — The Variational Approach to Fracture, Journal of Elasticity, 91 (2008), pp. 5-148.

[17] B. Bourdin, C. J. Larsen, and C. L. Richardson, A time-discrete model for dynamic fracture based on crack regularization, International Journal of Fracture, 168 (2011), pp. 133-143.

[18] B. Bourdin, J.-J. Marigo, C. Maurini, And P. Sicsic, Morphogenesis and Propagation of Complex Cracks Induced by Thermal Shocks, Physical Review Letters, 112 (2014), p. 014301.

[19] A. Braides, Approximation of free-discontinuity problems, Springer-Verlag, 1998.

[20] M. G. Callens, L. Gorbatikh, and I. Verpoest, Ductile steel fibre composites with brittle and ductile matrices, 61 (2014), pp. 235-244.

[21] J.-I. Choi, B. Y. Lee, R. Ranade, V. C. Li, Y. Lee, C. Composites, and N. York, Ultra-high-ductile behavior of a polyethylene fiber-reinforced alkali-activated slag-based composite, Cement and Concrete Composites, 70 (2016), pp. 153-158.

[22] J.-L. Clément, Interface acier-beton et comportement des structures en beton arme: caracterisation, modelisation, PhD thesis, Université Paris VI, 1987.

[23] V. Crismale, G. Lazzaroni, and G. Orlando, Cohesive fracture with irreversibility: quasistatic evolution for a model subject to fatigue, Preprint SISSA;40/2016/MATE, (2016).

[24] G. Czél, M. Jalalvand, and M. R. Wisnom, Design and characterisation of advanced pseudo-ductile unidirectional thin-ply carbon/epox - glass/epoxy hybrid composites, Composite Structures, 143 (2016), pp. 362-370.

[25] G. CzÉL And M. R. Wisnom, Demonstration of pseudo-ductility in high performance glass/epoxy composites by hybridisation with thin-ply carbon prepreg, Composites Part A: Applied Science and Manufacturing, 52 (2013), pp. 23-30.

[26] M. F. S. F. de Moura, R. Fernandes, F. G. A. Silva, and N. Dourado, Mode II fracture characterization of a hybrid cork/carbon-epoxy laminate, Composites Part B: Engineering, 76 (2015), pp. 44-51.

[27] G. Del Piero, One-Dimensional Ductile-Brittle Transition, Yielding, and Structured Deformations, in IUTAM Symposium on Variations of Domain and Free-Boundary Problems in Solid Mechanics: Proceedings of the IUTAM Symposium held in Paris, France, 22-25 April 1997, P. Argoul, M. Frémond, and Q. S. Nguyen, eds., Springer Netherlands, 1999, pp. 203-210.

[28] E. Esmaeeli and J. Barros, Flexural strengthening of RC beams using Hybrid Composite Plate (HCP): Experimental and analytical study, Composites Part B: Engineering, 79 (2015), pp. 604-620.

[29] G. A. Francfort And J.-J. Marigo, Revisiting brittle fracture as an energy minimization problem, Journal of the Mechanics and Physics of Solids, 46 (1998), pp. 1319-1342.

[30] F. Freddi And M. FrÉmond, Damage in domains and interfaces: a coupled predictive theory, Journal of Mechanics of Materials and Structures, 1 (2006), pp. 1205-1233.

[31] F. Freddi AND F. IURlano, Numerical insight of a variational smeared approach to cohesive fracture, Journal of the Mechanics and Physics of Solids, 98 (2017), pp. 156-171. 
[32] F. Freddi and G. Royer-Carfagni, Regularized variational theories of fracture: A unified approach, Journal of the Mechanics and Physics of Solids, 58 (2010), pp. 11541174 .

[33] — Variational fracture mechanics to model compressive splitting of masonry-like materials, Annals of Solid and Structural Mechanics, 2 (2011), pp. 57-67.

[34] _ Plastic Flow as an Energy Minimization Problem. Numerical Experiments, Journal of Elasticity, 116 (2014), pp. 53-74.

[35] — Phase-field slip-line theory of plasticity, Journal of the Mechanics and Physics of Solids, (2016).

[36] F. FRedd And E. SACCO, An interface damage model accounting for in-plane effects, International Journal of Solids and Structures, 51 (2014), pp. 4230-4244.

[37] H. Fukunaga, T. Chou, And H. Fukuda, Strength of Intermingled Hybrid Composites, Journal of Reinforced Plastics and Composites, 3 (1984), pp. 145-160.

[38] J. D. Fuller, M. Jalalvand, and M. R. Wisnom, Combining fibre rotation and fragmentation to achieve pseudo-ductile CFRP laminates, Composite Structures, 142 (2016), pp. $155-166$.

[39] J. D. Fuller And M. R. Wisnom, Damage suppression in thin ply angle-ply carbon/epoxy laminates, in 19th Int conf COMPOS Mater, Montreal, 2013, pp. 1-9.

[40] N. F. Grace, W. F. Ragheb, and G. Abdel-Sayed, Flexural and shear strengthening of concrete beams using new triaxially braided ductile fabric, ACI Structural Journal, 100 (2003), pp. 804-814.

[41] S. R. Hallett, Numerical Investigation of Progressive Damage and the Effect of Layup in Notched Tensile Tests, Journal of Composite Materials, 40 (2005), pp. 1229-1245.

[42] B. Halphen and Q. S. Nguyen, Generalized Standard Materials, Journal de Mécanique, 14 (1975), pp. 39-63.

[43] M. Jalalvand, G. Czél, J. D. Fuller, M. R. Wisnom, L. P. Canal, C. D. GONZÁLEZ, AND J. LLORCA, Energy dissipation during delamination in composite materials - An experimental assessment of the cohesive law and the stress-strain field ahead of a crack tip, Composites Science and Technology, 134 (2016), pp. 115-124.

[44] M. Jalalvand, G. CzÉL, And M. R. Wisnom, Numerical modelling of the damage modes in UD thin carbon/glass hybrid laminates, Composites Science and Technology, 94 (2014), pp. 39-47.

[45] — Damage analysis of pseudo-ductile thin-ply UD hybrid composites - A new analytical method, Composites Part A: Applied Science and Manufacturing, 69 (2015), pp. 83-93.

[46] C. P. Jiang, X. F. Wu, J. Li, F. Song, Y. F. Shao, X. H. Xu, and P. Yan, A study of the mechanism of formation and numerical simulations of crack patterns in ceramics subjected to thermal shock, Acta Materialia, 60 (2012), pp. 4540-4550.

[47] G. Lancioni and G. Royer-Carfagni, The Variational Approach to Fracture Mechanics. A Practical Application to the French Panthéon in Paris, Journal of Elasticity, 95 (2009), pp. 1-30. 
[48] A. A. León Baldelli, J. F. Babadjian, B. Bourdin, D. Henao, and C. Maurini, A variational model for fracture and debonding of thin films under in-plane loadings, Journal of the Mechanics and Physics of Solids, 70 (2014), pp. 320-348.

[49] A. A. León Baldelli, B. Bourdin, J.-J. Marigo, and C. Maurini, Fracture and debonding of a thin film on a stiff substrate: analytical and numerical solutions of a onedimensional variational model, Continuum Mechanics and Thermodynamics, 25 (2012), pp. 243-268.

[50] A. A. León Baldelli, C. Maurini, And K. Pham, A gradient approach for the macroscopic modeling of superelasticity in softening shape memory alloys, International Journal of Solids and Structures, 52 (2015), pp. 45-55.

[51] T. LI, Gradient-Damage Modeling of Dynamic Brittle Fracture: Variational Principles and Numerical Simulations, PhD thesis, École Polytechnique, 2016.

[52] T. Li and J.-J. Marigo, Crack Tip Equation of Motion in Dynamic Gradient Damage Models, Journal of Elasticity, 127 (2017), pp. 25-57.

[53] P. F. Liu, Z. P. Gu, Y. H. Yang, and X. Q. Peng, A nonlocal finite element model for progressive failure analysis of composite laminates, Composites Part B: Engineering, 86 (2016), pp. 178-196.

[54] A. Logg, K.-A. Mardal, And G. Wells, Automated Solution of Differential Equations by the Finite Element Method: The FEniCS Book, (2012).

[55] J.-J. Marigo, C. Maurini, and K. Pham, An overview of the modelling of fracture by gradient damage models, Meccanica, 51 (2016), pp. 3107-3128.

[56] C. Miene, S. Teichtmeister, and F. Aldakheel, Phase-field modelling of ductile fracture: a variational gradient-extended plasticity-damage theory and its micromorphic regularization., Philosophical transactions. Series A, Mathematical, physical, and engineering sciences, 374 (2016).

[57] A. Mielke, A Mathematical Framework for Generalized Standard Materials in the RateIndependent Case, in Multifield Problems in Solid and Fluid Mechanics, R. Helmig, A. Mielke, and B. Wohlmuth, eds., vol. 28 of Lecture Notes in Applied and Computational Mechanics, Springer Berlin / Heidelberg, 2006, pp. 399-428.

[58] A. Mielke And T. RoubíčEK, Rate-Independent Systems: Theory and Application, Springer, 2015.

[59] R. Mishra, J. Militky, N. Gupta, R. Pachauri, and B. Behera, Modelling and simulation of earthquake resistant $3 D$ woven textile structural concrete composites, Composites Part B: Engineering, 81 (2015), pp. 91-97.

[60] H. Nguyen, H. Mutsuyoshi, and W. Zatar, Hybrid FRP-UHPFRC composite girders: Part 1 - Experimental and numerical approach, Composite Structures, 125 (2015), pp. 631652.

[61] I. Novak and L. Truskinovsky, Nonaffine response of skeletal muscles on the 'descending limb', Mathematics and Mechanics of Solids, 20 (2015), pp. 697-720. 
[62] _ Segmentation in cohesive systems constrained by elastic environments Subject Areas :, Philosophical Transactions of the Royal Society of London A: Mathematical, Physical and Engineering Sciences, 375 (2017).

[63] T. Okabe, M. Nishikawa, And N. TAKedA, Numerical modeling of progressive damage in fiber reinforced plastic cross-ply laminates, Composites Science and Technology, 68 (2008), pp. 2282-2289.

[64] H. PetryK, Incremental energy minimization in dissipative solids, Comptes Rendus Mecanique, 331 (2003), pp. 469-474.

[65] K. Pham And J.-J. Marigo, Approche variationnelle de l'endommagement : II. Les modèles à gradient, Comptes Rendus Mecanique, 338 (2010), pp. 199-206.

[66] _ Stability of Homogeneous States with Gradient Damage Models: Size Effects and Shape Effects in the Three-Dimensional Setting, Journal of Elasticity, 110 (2012), pp. 6393.

[67] K. Pham, J.-J. Marigo, and C. Maurini, The issues of the uniqueness and the stability of the homogeneous response in uniaxial tests with gradient damage models, Journal of the Mechanics and Physics of Solids, 59 (2011), pp. 1163-1190.

[68] B. V. Rangan, Fly Ash-Based Geopolymer Concrete, in International Workshop on Geopolymer Cement and Concrete, 2010, pp. 68-106.

[69] Y. Swolfs, L. Gorbatikh, And I. Verpoest, Fibre hybridisation in polymer composites: A review, Composites Part A: Applied Science and Manufacturing, 67 (2014), pp. 181-200.

[70] Y. Swolfs, Y. Meerten, P. Hine, I. Ward, I. Verpoest, and L. Gorbatikh, Introducing ductility in hybrid carbon fibre/self-reinforced composites through control of the damage mechanisms, Composite Structures, 131 (2015), pp. 259-265.

[71] W. Weicheng Cui, M. R. Wisnom, and M. Jones, An Experimental and Analytical Study of Delamination of Unidirectional Specimens with Cut Central Plies, Journal of Reinforced Plastics and Composites, 13 (1994), pp. 722-739.

[72] R. Wetherhold And F. Lee, Shaped ductile fibers to improve the toughness of epoxymatrix composites, Composites Science and Technology, 61 (2001), pp. 517-530.

[73] J. Zhang, Y. Lu, L. He, L. Yang, AND Y. Ni, Modeling progressive interfacial debonding of a mud-crack film on elastic substrates, Engineering Fracture Mechanics, 177 (2017), pp. 123-132. 